\title{
Solids volume fraction measurements on riser flow using a temporal-histogram based DIA method
}

Citation for published version (APA):

Carlos Varas, A. E., Peters, E. A. J. F., Deen, N. G., \& Kuipers, J. A. M. (2016). Solids volume fraction measurements on riser flow using a temporal-histogram based DIA method. AIChE Journal, 62(8), 2681-2698. https://doi.org/10.1002/aic.15243

\section{Document license:}

TAVERNE

DOI:

10.1002/aic.15243

Document status and date:

Published: 01/08/2016

\section{Document Version:}

Publisher's PDF, also known as Version of Record (includes final page, issue and volume numbers)

\section{Please check the document version of this publication:}

- A submitted manuscript is the version of the article upon submission and before peer-review. There can be important differences between the submitted version and the official published version of record. People interested in the research are advised to contact the author for the final version of the publication, or visit the $\mathrm{DOI}$ to the publisher's website.

- The final author version and the galley proof are versions of the publication after peer review.

- The final published version features the final layout of the paper including the volume, issue and page numbers.

Link to publication

\section{General rights}

Copyright and moral rights for the publications made accessible in the public portal are retained by the authors and/or other copyright owners and it is a condition of accessing publications that users recognise and abide by the legal requirements associated with these rights.

- Users may download and print one copy of any publication from the public portal for the purpose of private study or research.

- You may not further distribute the material or use it for any profit-making activity or commercial gain

- You may freely distribute the URL identifying the publication in the public portal.

If the publication is distributed under the terms of Article 25fa of the Dutch Copyright Act, indicated by the "Taverne" license above, please follow below link for the End User Agreement:

www.tue.nl/taverne

Take down policy

If you believe that this document breaches copyright please contact us at:

openaccess@tue.nl

providing details and we will investigate your claim. 


\title{
Solids Volume Fraction Measurements on Riser Flow Using a Temporal-Histogram Based DIA Method
}

\author{
A. E. Carlos Varas, E. A. J. F. Peters, N. G. Deen, and J. A. M. Kuipers \\ Dept. of Chemical Engineering and Chemistry, Multiphase Reactors Group, Eindhoven University of Technology, \\ Eindhoven, The Netherlands \\ DOI 10.1002/aic.15243 \\ Published online April 14, 2016 in Wiley Online Library (wileyonlinelibrary.com)
}

\begin{abstract}
In this article we introduce a temporal histogram-based method for digital image analysis (DIA) of pseudo-2D fluidized bed risers. This method enables an accurate whole field measurement of the solids volume fraction in lab-scale pseudo$2 D$ riser flows by successfully removing image imperfections and merely accounting for the particles' intensity. Moreover, the new correlation between normalized intensity and solids volume fraction that is proposed in this work enables a quantitative approach for solids concentration measurements by DIA techniques. This technique can be easily adjusted with experimental settings and shows a great stability against adverse imaging conditions. The combination of this parameter-free method with particle image velocimetry under riser flow conditions has been successfully applied, enabling the experimental acquisition of full field hydrodynamic data. (c) 2016 American Institute of Chemical Engineers AIChE J, 62: 2681-2698, 2016
\end{abstract}

Keywords: digital image analysis, particle image velocimetry, fluidization, cfb, riser hydrodynamics, multiphase flow

\section{Introduction}

Riser reactors are widely employed in industry to carry out chemical processes that require high gas-solid contacting efficiency and short gas residence times. Among these processes are fluid catalytic cracking, biomass and coal gasification, granulation and metal coating. ${ }^{1}$ The extensive utilization of risers in industrial processes has driven an unceasing investment to boost their operational productivity. However, their performance has traditionally been optimized by costly and time-consuming experiments. ${ }^{2}$ In addition, an in-depth knowledge of fast-fluidized bed hydrodynamics is required for an optimal design and scale-up of riser reactors. As a consequence, several experimental techniques have been developed over the past decades to study the circulation patterns of fluidized systems. ${ }^{3-5}$ Some intrusive techniques such as optical probes, endoscopes or hot wires have been widely employed in the past to collect cross-sectional porosity data of fluidized beds.

Optical probes have been employed by several authors to measure local particle concentration and velocity. ${ }^{6-9}$ For instance, Gidaspow et al. employed particle image velocimetry (PIV) to measure particle velocities and solid turbulent properties in a 3 -D circulating fluidized bed. ${ }^{8}$ This work was also combined with $\gamma$-ray densitometry to measure particle concentrations covering the radial direction of two axial positions, by inserting an optical probe at different lengths from the insertion port. More sophisticated optical techniques make use of

\footnotetext{
Correspondence concerning this article should be addressed to E. A. J. F. Peters at E.A.J.F.Peters@tue.nl.

(C) 2016 American Institute of Chemical Engineers
}

particle recognition methods to measure particles velocities in fluidized systems.

However, these optical techniques are invasive and are restricted to local measurements, without providing full-field experimental data under riser flow conditions. Other nonintrusive methods are gradually replacing invasive techniques, such as electrical capacitance tomography, ${ }^{10}$ laser doppler anemometry, ${ }^{11}$ phase doppler anemometry, ${ }^{12}$ magnetic resonance imaging ${ }^{13}$ or magnetic particle tracking ${ }^{14,15}$ or PIV. All these technologies can provide accurate results, where PIV is among the most reliable and economical techniques. ${ }^{14}$ For particulate systems the PIV technique has been applied to pseudo-2D bubbling fluidized beds to obtain full-field hydrodynamic data, ${ }^{16}$ but the sole employment of this technique does not provide a full description of the solids motion. Link et al. 2005 were the first authors to collect full-field experimental data on solids mass flux measurements by a combination of PIV (for velocity fields) and Digital Image Analysis (DIA) for solids volume fraction data. ${ }^{17}$ This DIA technique requires high-quality normalized images to accurately quantify solids volume fraction data of a pseudo-2D fluidized system. Laverman et al. made use of local image filters to increase the quality of the collected images, which in a later stage were converted into porosity fields by means of a linear correlation. $^{18}$

Since current full-field DIA techniques can only be applied to bubbling fluidized beds, we were prompted to develop a novel DIA post-processing method, which in combination with PIV, provides complete hydrodynamic data sets of pseudo-2D riser flows. The DIA technique estimates solids volume fraction data from experimentally obtained visual images. Van Buijtenen et al. related solids volume fractions to normalized intensity data using an equation that involves two 
fitting parameters that were calibrated with the solids weight, ${ }^{19}$ which is a known parameter in a bubbling fluidized bed. They used computer-generated synthetic images from CFD-DEM simulations of a fluidized bed to find the proper parameters for the correlation. De Jong et al. further researched the influence of other experimental parameters like the particle diameter $\left(d_{p}\right)$, system depth and light intensity with synthetic images as well. ${ }^{20}$ It was claimed that the solids volume fraction of a bubbling fluidized bed could be accurately related to the image intensity through a correlation with constants that are fixed by the fluidization conditions. In this work, we introduce the new DIA post-processing method that provides reliable solids volume fraction data of a riser by means of a temporal histogrambased DIA algorithm. This technique can be complemented with PIV to fully characterize the circulation pattern of a riser.

Conventional spatial DIA methods require homogeneous lighting and a clear contrast between particles and background to obtain accurate hydrodynamic data. The presence of inhomogeneous illumination or a noisy background are unwanted characteristics of a snapshot to successfully estimate the solids volume fraction of a fluidized system. The lighting of an object depends not only on the number of light sources used, but also on the illuminance of each light source as well as their respective distances to the object. Thus, an even light intensity of a specific object can be quite unfeasible if a relatively large area needs to be illuminated with a couple of LED light sets. This will result in decreased lighting quality due to the presence of shadows, whose area could be considerably larger than the size of a single particle. For instance, in a recorded riser section of $0.8 \mathrm{~m}$ height, regions close to the walls are very susceptible to shadows as well as to spots in the stainless steel back plate originating from its manufacturing process. In this scenario, there are many unwanted details that are captured by the high-resolution camera, making their removal essential. Casleton et al. analyzed the influence of specular reflections on particle quantification of a circulating fluidized bed, ${ }^{21}$ while Yang et al. employed a reference plate of unspecified dimensions to ensure constant illumination conditions throughout the recorded section of a circulating fluidized bed and avoid inaccuracies in cluster solids holdup measurements. ${ }^{22}$ However, to obtain a homogeneous light field over a relatively large recording area is quite unfeasible.

An important class of method that attempt to remove these unwanted details are background subtraction methods. These methods have been widely employed to improve the image quality in computer vision systems, surveillance cameras, medical imaging and other disciplines. The algorithms have evolved from off-line methods; where purely temporal methods are applied; to spatio-temporal filters, where the background is adapted in time and learns new changes in the image environment. $^{23}$ Other image segmentation algorithms have been developed to isolate foreground objects to obtain binary images of moving objects for instantaneous image recording. More complex image recognition patterns have been also employed to compute the motion of an image body between consecutive images, as in PIV algorithms.

Image segmentation has been previously applied to distinguish particles in fluidized systems. However, these investigations make use of spatial filters. Chen et al. reported the average particle diameter in a bubbling fluidized system ${ }^{24}$ and Wei et al. analyzed the dispersion of tracer particles in a circulating fluidized bed. ${ }^{25}$ These techniques employ thresholding techniques and local image filters; and lack a clear methodol- ogy to quantify particle concentrations in gas-solid flows by image processing techniques. Other authors performed fullfield measurements of solids volume fraction on lab scale units under bubbling fluidization conditions. To enhance the quality of the post-processed image, filtering techniques have been employed $^{18}$ to remove image anomalies that could significantly decrease the accuracy of the solids volume fraction measurements. ${ }^{26,27}$ However, local image filtering techniques, which are used in the current full-field DIA methods, have a blurring effect on the image. ${ }^{28}$ The employment of these filters can distort the image information to such an extent that some particles are undetected. They therefore lead to significant inaccuracies in the solids volume fraction measurements if this process occurs frequently.

The aim of this article is to define a clear methodology to perform full-field experimental measurements of solids volume fraction on pseudo-2D fluidized systems. This technique can generate a generous amount of experimental references for more thorough validation procedures for CFD models.

Thus, we present a new DIA post-processing method that enhances the reliability of determining axial and crosssectional solids volume fraction data on pseudo-2D risers. The present paper reports a DIA post-processing technique that enables full-field solid concentration measurements. Moreover, the technique removes shadows and image imperfections without requiring the use of spatial image filters which distort the quality of the solids volume fraction measurements. This so-called temporal histogram method (THM) automatically overcomes lighting issues and does not need any calibration with a constant solids content that is inherently changing in a riser. In this way, applicability of DIA is extended to measuring solids volume fraction data in pseudo-2D risers of lab scale.

To test the new method, we make use of CFD-DEM simulations to generate "synthetic images." Particle positions from the simulations are used to create an image, with imperfections such as noise and shadows modeled. The goal is to create output that closely resembles real experimental images to obtain an accurate correlation. Synthetic images are post-processed and are related to instantaneous solids volume fraction data from simulations. This approach is similar to that used by Van Buijtenen et al. and De Jong et al., who correlated these data by means of a function with two fitting parameters. Previous full-field DIA techniques were developed for experiments, where lighting conditions are ideal and therefore are sensitive to lighting conditions. An important contribution of the technique presented in this article is that, we implemented subpixel intensity to obtain a closer resemblance to experimental images. Furthermore, we employ a temporal filter that provides intensity data that are directly proportional to the pixel coverage by particle phase; and $A$ and $B$ values are insensitive to image quality issues. The robustness of this new DIA method is tested under different imaging conditions to ensure reliable solids volume fraction data even under non-optimal lighting conditions of the illuminated section. The main features of the different DIA techniques, will be assessed to perform a qualitative comparison between post-processed images by different methods. Besides, the performance of THM will be compared to the full-field DIA method developed by De Jong et al. to evaluate the accuracy of these two methods to measure solids volume fraction in fluidized systems. An accurate correlation between solids volume fraction and image intensity will be provided, and its dependence on imagerelated properties is quantified as well. 


\section{Methodology}

\section{Instruments}

Experimental Setup. DIA experiments were carried out on a circulating fluidized bed that consists of a cylindrical downcomer to recirculate solid particles throughout the system, and a pseudo-2D riser (see Figure 1). In the riser unit, solid and gas phases co-currently flow upwards to the top of the riser, where a cyclone is used to obtain the gas-solid separation. A pseudo-2D riser with dimensions $1500 \times 70 \times 6 \mathrm{~mm}$ (height $\times$ width $\times$ depth), was employed to collect experimental images and compare the performance of THM with conventional DIA post-processing methods.

This rectangular chamber has a $1 \mathrm{~cm}$ thick glass front wall to ease visual access to its interior and a stainless steel back plate to allow an operating pressure of 2 bar. Black Geldart D particles of $0.8-0.9 \mathrm{~mm}$ diameter and $2500 \mathrm{~kg} / \mathrm{m}^{3}$ density, were fluidized with a compressed air stream. The gas was evenly distributed by means of a porous stainless steel plate at the bottom of the riser. To collect experimental images of the riser flow, four LED light sets were employed to illuminate the pseudo-2D riser. The images were recorded by a high resolution camera (LaVision ImagerproHS4M) that was placed at approximately $3 \mathrm{~m}$ from the experimental unit. The recordings were made at the top $0.8 \mathrm{~m}$ of the riser. Post-processed image data of the recorded experiment were generated by means of custom Matlab script.

Simulations. CFD-DEM simulations of a pseudo-2D riser with dimensions $900 \times 70 \times 6 \mathrm{~mm}$ (height $\times$ width $\times$ depth) were employed to generate synthetic images, which were used to perform the calibration and sensitivity analysis of the presented DIA method for determining solids volume fractions. The Eulerian-Lagrangian model solves the Navier-Stokes and continuity equations to describe the gas phase dynamics, while the solids movement is described by Newton's second law of motion. The gas-solid interactions are coupled by the Beetstra drag force correlation, ${ }^{29}$ whereas the collision parameters correspond to glass beads properties ${ }^{30}$ previously reported by Hoomans et al. Further details of the simulation settings are specified in Table 1 .

Prescribed atmospheric pressure, inflow velocity and noslip boundary conditions, respectively, were imposed at the top, bottom plane, and the side walls of the simulation domain. The particles were fed into the system at random positions in the bottom $\mathrm{X}-\mathrm{Y}$ plane of the simulation domain, and left at the top of the system. The superficial velocity and solids inflow parameters were such that dense and dilute regions were created. This is important because, to correlate postprocessed synthetic images and solids volume fraction data from simulations, the whole solids volume fraction range (from 0 to maximum packing fraction $\approx 0.6$ ) is needed.

CFD-DEM Synthetic Images. Grayscale digital images are numerical representations of the light intensity of a recorded object. Given a grayscale digital image of 8 bits of a fluidized system, the background of which is totally white reaching the maximum intensity (255) whereas the particle is so dark that the minimum intensity value of the 8-bit image (0) is reached. Thus, minimum intensity values will only be given in pixels that are fully covered by particles, while totally empty pixels will reach maxima intensities. However, pixels that are partly covered by particles provide an interpolated value between minimum and maximum intensities in a range of 256 different shades proportional to the degree of pixel coverage.

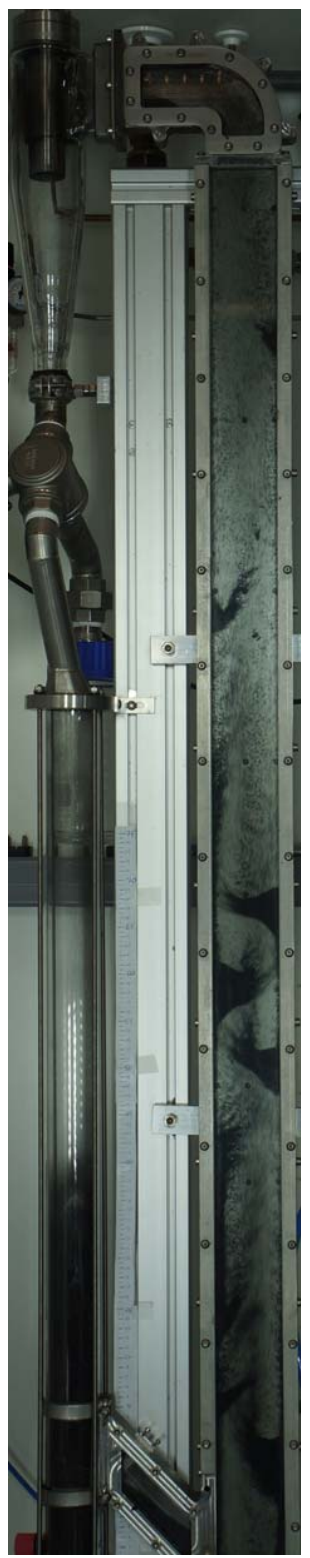

Figure 1. Experimental setup.

[Color figure can be viewed in the online issue, which is available at wileyonlinelibrary.com.]

The gray values of a digital image are proportional to the incident light intensity. Our particles are large compared to the wavelength of visual light. Therefore the measured intensities are assumed to not be influenced by diffraction phenomena. According to Beer-Lambert law, the material transmittance of an object decays exponentially with the light path depth and the material light absorbance. However, the particles used are so opaque that transmittance is negligible compared to reflection and absorbance. The particles are coated black such that most incident light will be absorbed and little reflected. For the material of the back plate this is different. This is lightly colored such that a large portion of the incident light is diffusely reflected from it. Since we use lamps at different angles we assume that, at least for not too high solids volume fractions, the back plate is always well illuminated.

If a particle is in view its main influence is the blockage of the light coming from the background. Consequently, we assume that particles are always darker than the riser 

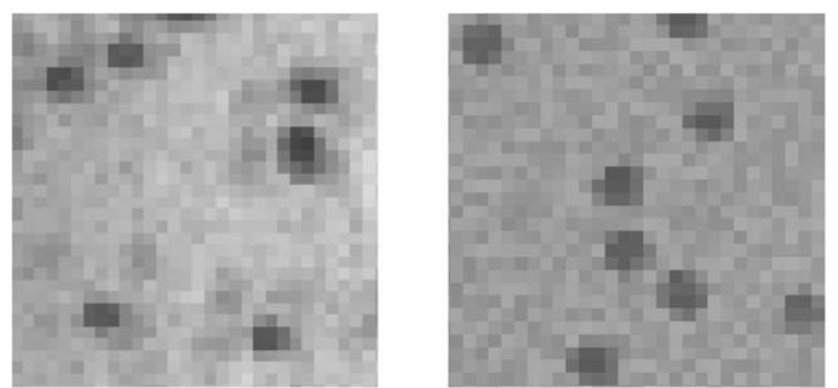

Figure 2. Left: $0.8-0.9 \mathrm{~mm}$ black particles from experiments. Right: A synthetic image of black $1.0 \mathrm{~mm}$ particles.

background as can be perceived in Figure 2. Thus, this method is developed for particles that have a higher absorbance than the background, and its application to reflecting/transparent particles remains to be investigated. The gray-scale recorded by a pixel of the camera, in our case, is thus mainly determined by two factors, one is the (partial) blockage of the illuminated background by particles. The second factor is local illumination conditions. Our method consists of, as much as possible, eliminating the influence of this second factor and thus measuring the "degree of pixel blockage." The next step is to correlate this filtered or normalized light intensity to the local solids volume fraction.

In the experiments, the particles are only a few pixels in size. This means that a substantial portion of the pixels will be partly covered. If, in the synthetic images, we give pixels a value only depending on whether it is covered "yes" or "no" this gives a poor representation of the edges of the particles, e.g., generating for instance a stair-case visual effect. ${ }^{31}$ These type of distortions are commonly called spatial aliasing. To amend this visual effect, an anti-aliasing technique called subpixel sampling (with $8 \times 8=64$ subpixels) is implemented to render the total pixel intensity as:

$$
I_{\text {pixel }}=\frac{n}{N} I_{\text {particle }}+\left(\frac{1-n}{N}\right) I_{\text {background }}
$$

where $n$ is the number of subpixels that are occupied by the circular area of a particle with diameter equal to $d_{p}$, and $N=64$ is the total number of subpixels that are sampled. This is an old and efficient technique that avoids spatial aliasing of rendered objects in grayscale digital images. ${ }^{23}$ This procedure makes that the grayscale of a pixel in synthetic image is linearly dependent on the fraction of the pixel that is covered by a particle. Thus, subpixel sampling faithfully mimics the image of a particle that this is registered by a CCD camera. ${ }^{32}$

A homemade script is used to read the location and radii of the particles from the simulation data files to assign an intensity value to the new image. While background intensities would be fixed at the neighborhood of each particle location, the particle area per pixel is estimated by subpixel sampling to assign a proportional intensity value. In Figure 2, the qualitative resemblance of the synthetic and the experimental image pattern is clear. Each particle is represented by its core intensity when this is fully occupying a pixel; while around its center, there are intermediate values between background's and core particle intensities. Thus, we conclude that a similar particle rendering to real experiments is achieved in these synthetic images through the implementation of subpixel sampling.

Shadows, light gradients, and background spots are also generated to test the robustness of the post processing method 


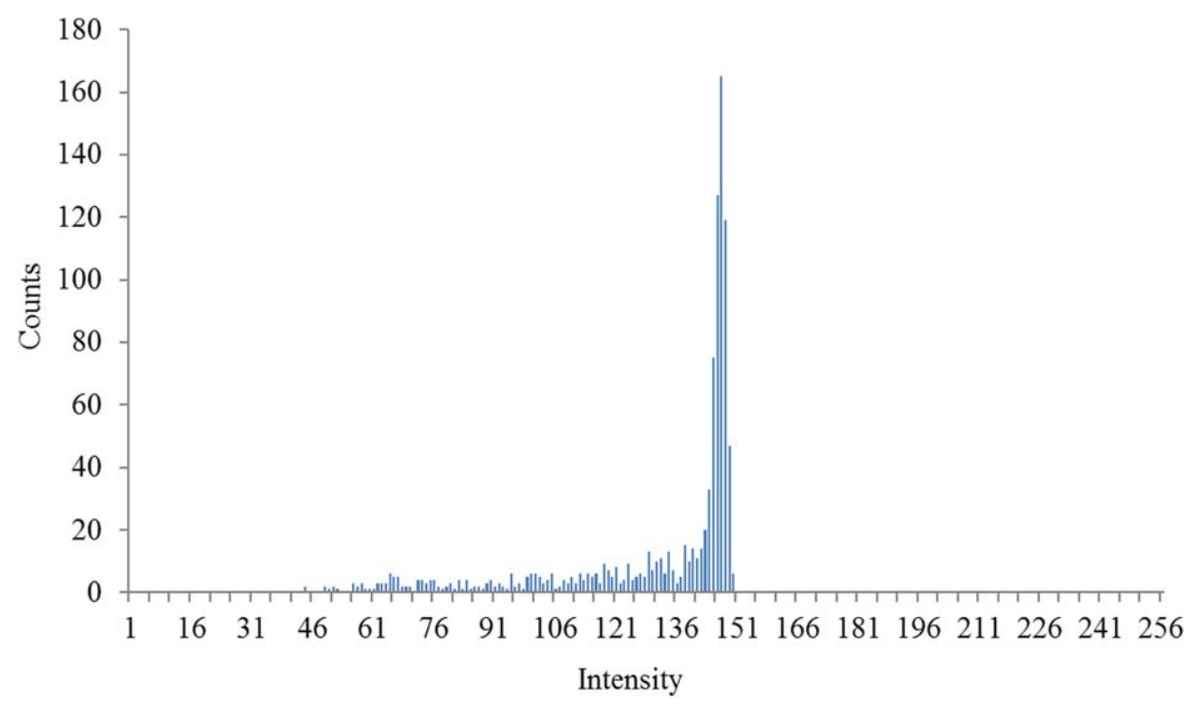

Figure 3. Temporal data histogram of an experimental image for a single-pixel.

[Color figure can be viewed in the online issue, which is available at wileyonlinelibrary.com.]

under different kinds of image distortion factors (whose details are further explained in Appendix C). These image imperfections are found to be very troublesome for local spatial image filtering techniques, while it will be shown (for our application) that the new THM method is insensitive to these factors due to its single-pixel normalization.

\section{Temporal histogram DIA method}

Normalization. In a video recording of riser flow, the gray value of an individual pixel will be, most of the time, corresponding to the light intensity of the part of the riser's backplate in its field of view. This value might fluctuate a little bit in time, e.g., due to variations in the lighting, electronic noise in the camera or shadows/reflections of nearby particles. Only when a particle passes by will the gray value be markedly different. The exact value recorded at this moment in time depends on the coverage of the pixel by the particle (partly or full), and the light intensity from the particle. The intensity will be influenced by the lighting of the part of the bed viewed, but also by shadows or reflections from, e.g., side walls and other particles.

By making a histogram in time for each individual pixel, information is obtained such as the minimum light intensity and the maximum light intensity. This minimum and maximum intensity are identified by, respectively, a fully covered and uncovered state of the pixel. Note that for this to be true, a recording should be long enough such that, each pixel has been at least once fully covered by a particle. Therefore a histogram in time for an individual pixel can be used to normalize the value to perform particle detection, where a value 0 means only background, a value 1 full coverage, and a value in between partial coverage of the pixel by a particle.

The images of a video will be processed twice. In the first pass, a histogram is built for each pixel. In a second pass, the information of the pixel histograms is used to renormalize the snapshots. In the results section, we will show that by means of this temporal normalization method the effects due to uneven lighting and shadows will be largely filtered out. Therefore there is no need for spatial filtering and there is no loss of definition due to blurring, etc.

The new THM method overcomes the issues of spatial filtering methods. Its novelty remains in the application of a tem- poral histogram-based post-processing method for particle quantification purposes. The intensity of each pixel is normalized over the intensity difference between the minimum and maximum grayscales of this pixel. Thus, when a particle partly covers a pixel, its intensity will be normalized over the width of its respective temporal histogram, computing in this way values ranging between 0 and 1 depending on the pixel intensity.

When only the minimum and maxima values for each pixel needs to be known, it is actually not necessary to build up a full histogram, in the first pass through the recording only the minimum and maximum values for each pixel needs to be updated and stored. However, full histogram information can be useful for more advanced processing, like computing a customized Otsu threshold. The histogram shape is qualitatively the same for all pixels, showing a high peak at maxima intensities and a decreasing tail toward minima intensities. Since the background intensity frequency is much higher than a particle's, the tail may not be clearly visible in histograms of pixels located at more dilute areas of the domain. Therefore a pixel histogram obtained from the denser bottom section of the riser is shown in Figure 3. The peak at the gray level value of $I_{\max }(i, j)=145$ (8 bit-image) corresponds to the background. It has a finite width that is caused by small variations in light intensity and camera noise. The minimum value is: $I_{\min }(i, j)=44$. This level corresponds to a pixel fully covered by a particle and possibly lowered a bit due to fluctuations or shadows. Thus, the intensity of each pixel is normalized as:

$$
\varphi_{2 D}(i, j)=\frac{I_{\max }(i, j)-I(i, j)}{I_{\max }(i, j)-I_{\min }(i, j)}
$$

where $I_{\min }(i, j)$ and $I_{\max }(i, j)$, respectively, are the minimum and maximum intensities in the temporal histogram of the pixel under consideration.

Correlation 2D-3D Solids Volume Fraction. Once each picture is normalized, a suitable correlation is needed that translates these intensity values into solids volume fraction data. The solids volume fraction which will be indicated as $\varphi_{3 D}$. The notion of a local solids volume fraction only is useful if volumes containing several particles are considered. Therefore, we will consider windows of $8 \times 8$ pixels and compute 

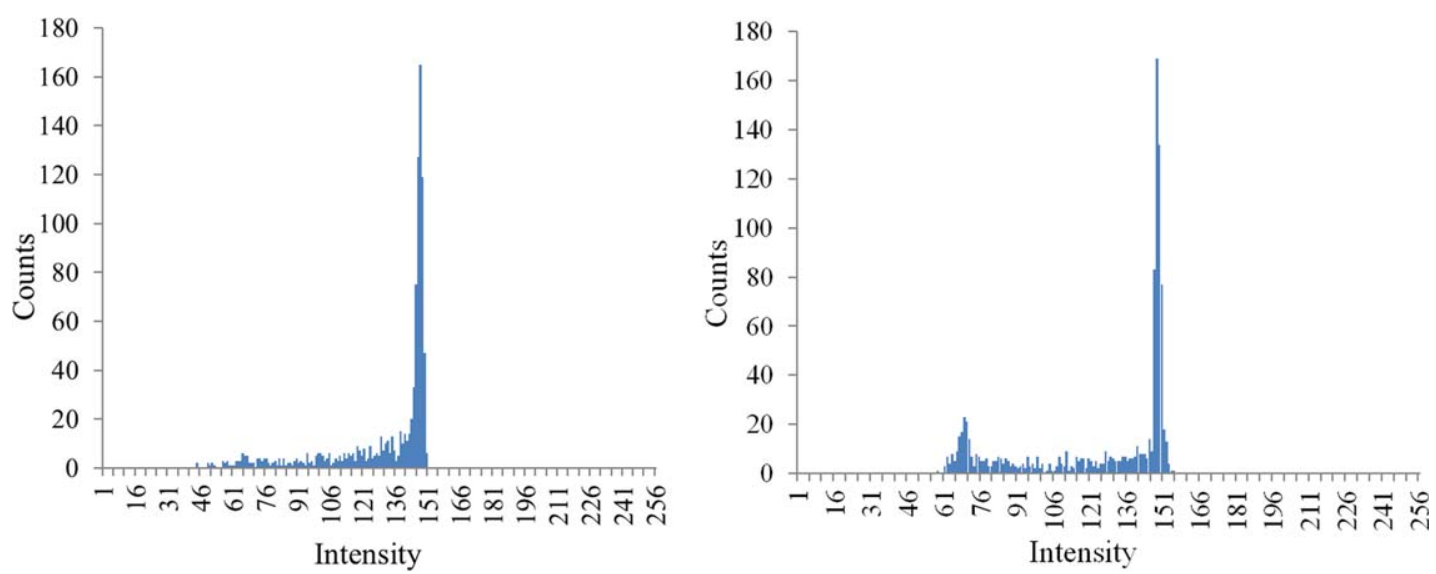

Figure 4. Left: Temporal intensity histogram of a pixel from an 8-bit image of pixels computed over 1000 experimental images on riser flow. Right: Temporal intensity histogram of a pixel over 1200 synthetic images containing intensity fluctuations mimicking camera electronics noise.

[Color figure can be viewed in the online issue, which is available at wileyonlinelibrary.com.]

average normalized 2D intensities, $\varphi_{2 D}$, for each window. For very dilute systems where particle overlap in an image is minimal, one expects the averaged $2 \mathrm{D}$ normalized intensity, $\varphi_{2 D}$, and the solids volume fraction, $\varphi_{3 D}$, to be related as

$$
\varphi_{3 D}=\frac{N_{p}}{A D} \frac{\pi}{6} d_{p}^{3}=\frac{2}{3} \frac{d_{p}}{D} \frac{N_{p}}{A} \frac{\pi}{4} d_{p}^{2} \approx \frac{2}{3} \frac{d_{p}}{D} \varphi_{2 D}
$$

where $D$ is the bed depth and $d_{p}$ the particle diameter $\left(N_{p}\right.$ : number of particles, $A$ : bed area).

CFD-DEM simulations, using an in-house code of a pseudo-2D riser, were employed to generate synthetic images and relate the $2 \mathrm{D}$ intensity values to the solids volume fraction data from the simulations' output. First, data files were generated every $0.005 \mathrm{~s}$ from simulations, collecting a total of 1200 files with all the hydrodynamic information of the system. Next, the pixel-based information of each image $(2184 \times$ 168 pixels) was locally averaged into an $8 \times 8$-pixel data field of $273 \times 21$ windows. From each simulation, a total of around 191,000 data points are plotted to relate the normalized intensity values to solids volume fraction from simulation data. These data points are fitted to the functional form found below (fitting procedure is further explained in Appendix B):

$$
\varphi_{3 D}=A \cdot B \cdot \operatorname{arctanh}\left(\frac{\varphi_{2 D}}{B}\right)
$$

The variables are chosen such that $A$ is the linear coefficient near zero solids volume fraction, i.e., $\varphi_{3 D} \approx A \cdot \varphi_{2 D}$. We therefore expect that $A \approx\left(2 d_{p}\right) /(3 D)$. Parameter $B$ is the saturated value of $\varphi_{2 D}$, which is expected to equal 1 . It will be shown that the $A$ and $B$ constants have fixed values at a given $d_{p} / D$ ratio, independent of lighting conditions, etc. A clear achievement, compared to previous methods, is that THM does not need any calibration with the solids weight of the system, which is unknown under riser flow conditions. A sensitivity analysis of the model will be presented in the results section.

\section{Results and Discussion Image characterization}

General Features. Specific characteristics of conventional and novel DIA methods will be discussed in detail in this section. To fully convey the general features of these techniques, a general description of conventional DIA methods can be found in Appendix A. These methods, developed by Van Buijtenen et al. ${ }^{19}$ and De Jong et al. ${ }^{20}$, were calibrated by using synthetic images, where particles had a Gaussian internal profile distribution of the light intensity, reaching the highest intensity at the particle core and decreasing intensity toward its edges.

Another fundamental characteristic that was assumed in the generation of synthetic images by previous authors ${ }^{19,20}$, was the particle light intensity variation in the depth direction. In this way, particles closer to the front wall have a higher light intensity than those near the back wall. This effect could be assumed in real experiments if the particles were light colored and particles at front layers cast shadows on particles that are near the back of the system. However, this phenomenon does not occur in our experiments where the background is light colored and the particles are dark. Shadows cannot be distinguished on dark particles. Moreover, the internal intensity distribution of a particle, as observed in our visual recordings, does not occur due to any lighting effect, but simply due to the fact that interpolated intensity values are captured by the camera when a particle only partly covers a pixel. Thus, we assume that the internal intensity profile of a particle does not have a Gaussian distribution, but its edges are represented by partly occupied pixels. These pixels have interpolated values between that of an empty and fully covered pixel.

These observations tell that the models developed by Van Buijtenen et al. ${ }^{19}$ and De Jong et al. ${ }^{20}$ are not universal and only correspond to a specific image pattern that is different than the one we have in our experiments.

The images of the first experiments on our lab scale pseudo2D riser (see Figure 2) show a slightly noisy light background and dark spots at the positions of the particles. It can be observed that the particle center is darker than its edges due to partial pixel coverage of the particle. Furthermore, in our experiments, the particle intensity was found to be independent of the depth direction. All this indicates that the internal particle intensity profile is flat and independent of the depth location of the particle. So, in our synthetic images, the particle light intensity was kept constant through its internal projected area and all over the depth of the pseudo-2D system.

In Figure 4, a comparison between experimental and synthetic images shows a quantitative similarity between 


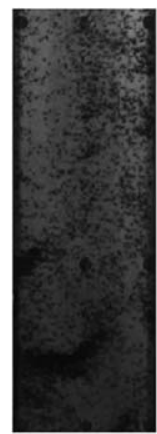

a

Figure 5. (a) Instantaneous experimental image. (b) Maxima intensity field. (c) Minima intensity field. (d) Contrast field.

synthetic and experimental images. To achieve this, a set of 1000 experimental and another set of 1200 synthetic images have been pre-processed to build temporal intensity histograms of a single pixel. The shape of the intensity temporal histogram conveys relevant information about the image pattern of both sets of images, giving quantitative information about the degree of dilution of the fluidized system at a particular pixel location. In this case, pixels located at the bottom of the riser were selected to have a similar frequency of particle and background intensities.

In both histograms shown in Figure 4, a high-peak at maxima intensity values is observed, which correspond to the background. Next to this peak, there is a decreasing slope to lower values, corresponding to intensities due to particle coverage. In the synthetic images this only happens when there is a partially covered pixel. Thus, we can confirm that the implementation of subpixel intensity on synthetic images achieves a quantitative correspondence to the images of our experiments.

It has to be noted that in experiments, the background intensity oscillates between 4 and 5 grayscales due to noise of the camera electronics. White noise of the camera electronics has been mimicked by adding fluctuating noise with a standard deviation of 4-5 gray levels. This effect can also be noticed in both histograms presented in Figure 4. Thus, we can assume that the shape of the temporal histogram on DIA experiments is mainly due to intermediate intensities that are perceived by the high resolution camera when pixels are partly covered by particles.

Contrast. In the synthetic images, it was assumed that regardless of the image imperfections, the particle intensity was always lower than the background intensity, assuming that the presence of shadows not only reduces the background's but also the particle's intensity. To confirm that this visual characteristic is also present in experiments, the image pattern needs to be further characterized and we should check that the particle light intensity field is significantly lower than the background light intensity field of our experimental setup. Thus, we register maxima and minima intensities to recon- struct, the background and particle intensities over the whole domain, obtaining qualitative information about the lighting conditions of our experimental setup. These intensity fields were obtained by acquiring intensity values over 1000 experimental images.

Minima and maxima intensity values were stored during an experiment to check that these values correspond to particle and background phases, respectively. In Figure 5, it can be observed that maxima intensity values exactly describe the background of the experimental unit, even capturing imperfection details due to its manufacturing. Whereas minima intensities, correspond to particle intensities. The contrast field is defined as the difference between the minima and the maxima intensity fields.

It can be observed that there is a noticeable intensity difference between the two fields, which reveals that both phases are distinguishable throughout the riser domain. Since the intensities of both phases are dependent on the location in the riser, it can be said that both are affected by external light conditions. This confirms that everywhere in the bed, the particle intensity is lower than the background intensity, which forms the basis for identifying the particles in our method (more details on Appendix C).

As mentioned the image contrast is defined in our case as the width of the temporal histogram, which corresponds to the intensity difference of the background and the intensity of a pixel which is fully covered by a particle. The temporal histograms are employed to normalize each pixel intensity, and therefore have an influence on the solids volume fraction quantification. As the contrast of an image can be different between experiments, this variable will also be evaluated in the sensitivity analysis of the proposed correlation.

\section{D-Intensity normalization: THM vs. conventional DIA}

In this section, the THM performance is compared to the performance of a previously reported full-field DIA method ${ }^{20}$ (detailed in Appendix A) under three different cases, of which the main characteristics are described in Table 2. These cases are investigated to draw conclusions about the quality of the temporal normalization procedure that was previously explained.

In case 1, 8-bit images with a substantial contrast were generated, i.e., " 150 " intensity for the background and " 0 " for the particle phase. The selected intensities mimic an experiment with ideal lighting conditions along the whole domain of the system, without shadows or background spots that could negatively influence the local thresholding techniques that are applied in conventional DIA methods.

When recording large areas, shadows and light gradients are frequently encountered throughout the illuminated experimental unit. In our case, when a pseudo-2D riser is recorded, not only shadows are present near the walls, but also there is a decreasing light intensity from the center of the riser to its edges, due to uneven lighting. These characteristics are captured in case 2 .

Table 2. Characteristics of Cases 1-3

\begin{tabular}{|c|c|c|c|c|c|}
\hline & Images source & Background intensity & Particle intensity & Shadows and spots & Subpixel intensity \\
\hline Case 1 & DPM & 255 & 105 & No & Yes \\
\hline Case 2 & DPM & $135-155$ & $115-130$ & Yes & Yes \\
\hline Case 3 & Experiments & $16-255^{\mathrm{a}}$ & $8-78^{a}$ & Yes & - \\
\hline
\end{tabular}

${ }^{a}$ The background and particle intensities are changing according to the illuminance of the riser location. For each case 1,200 images were processed. 


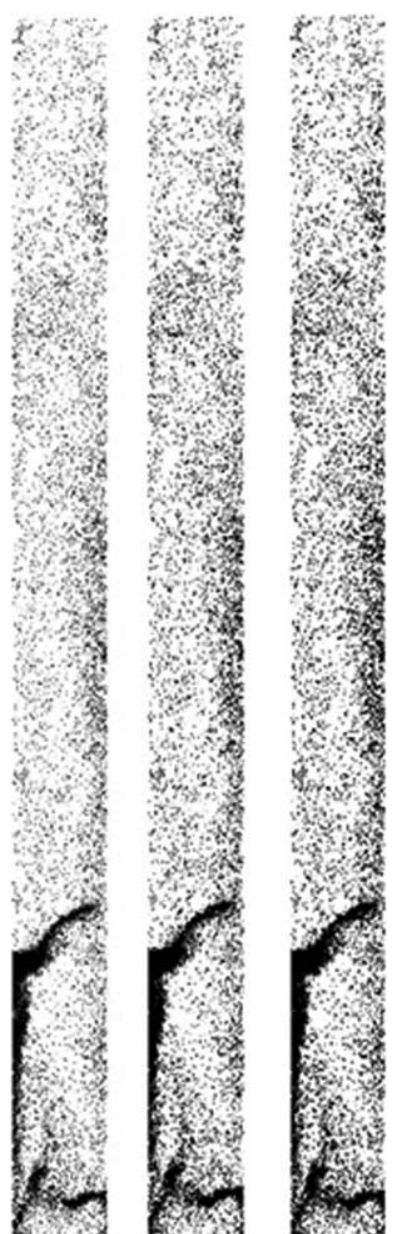

Figure 6. Left: Original image. Middle: normalized image obtained from conventional DIA method. Right: normalized image obtained from THM processing.

Images of case 2 have a considerably lower contrast than in case 1 , making the distinction between the phases more difficult. Moreover, a light gradient is generated along the whole domain to simulate the uneven lighting that is most likely present when such large areas are not evenly illuminated. Shadows close to the walls are also included to mimic a more realistic case and test the robustness of the normalization procedure of the novel DIA method against conventional local image filtering techniques.

Finally, case 3 presents images from an experiment conducted in a circulating fluidized bed of our laboratory, where light-related issues are found as they are commonly present in experiments.

Case 1. The normalization processing of this idealized case is trivial for both methods. The absence of shadows or background spots enables the synthesis of normalized images of high quality by both DIA techniques. In Figure 6, the relevant images are shown: the original synthetic image and two post-processed images, one normalized by conventional DIA method, and another by THM. This case illustrates how both, spatial and temporal normalization methods are effective for optimal lighting conditions.

It can be noticed that both post-processed images are almost identical to the original one, revealing that the quality of the normalized image is very high in both cases. Thus, the performance of both methods under excellent visualization conditions would be similar for Case 1 .

Case 2. In this case background noise and shadows are artificially generated on the synthetic image. Moreover, a light gradient is created along the image to mimic the illumination of such a large area. The original synthetic image is shown first in Figure 7, and next the post-processed images by conventional DIA and THM, respectively.

To have a normalized image of high quality, the image should only account for intensity values where the particles are present, while intensities generated by other external factors should be removed or minimized as much as possible to not quantify them when the 3D solids volume fraction is calculated from the intensity. Figure 7 clearly shows that the conventional DIA processing cannot handle poor lighting conditions well. Conversely, the THM method, removes nearly all perceptible imperfections of the image and just captures intensity values due to the particles presence. Since image imperfections are static throughout time, the temporal character of THM enables to only detect the particles intensity, which is the only dynamic information that changes over the whole set of post-processed images.

In Figure 7, the conventional DIA method with image filtering techniques is applied to improve the quality of the

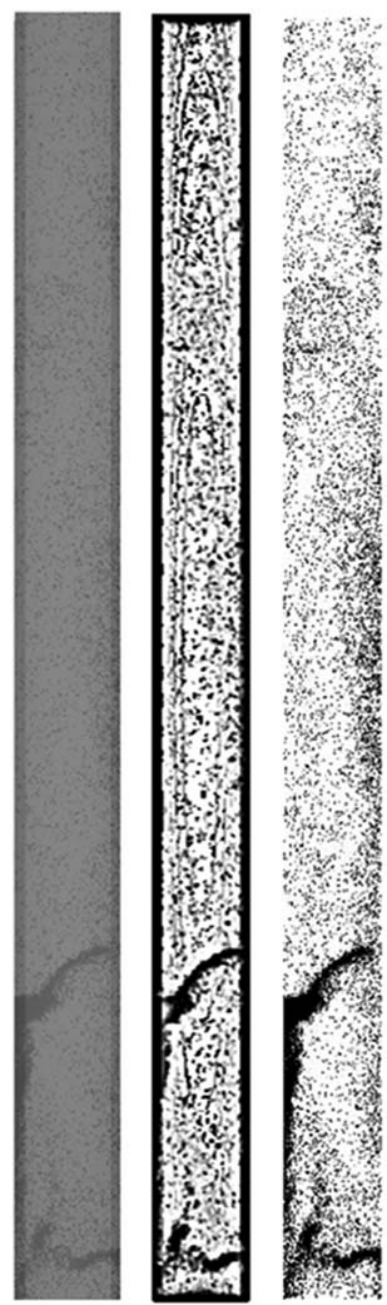

Figure 7. Case 2 post-processing. Left: Original image. Middle: normalized image obtained from conventional DIA method. Right: normalized image obtained from THM processing. 

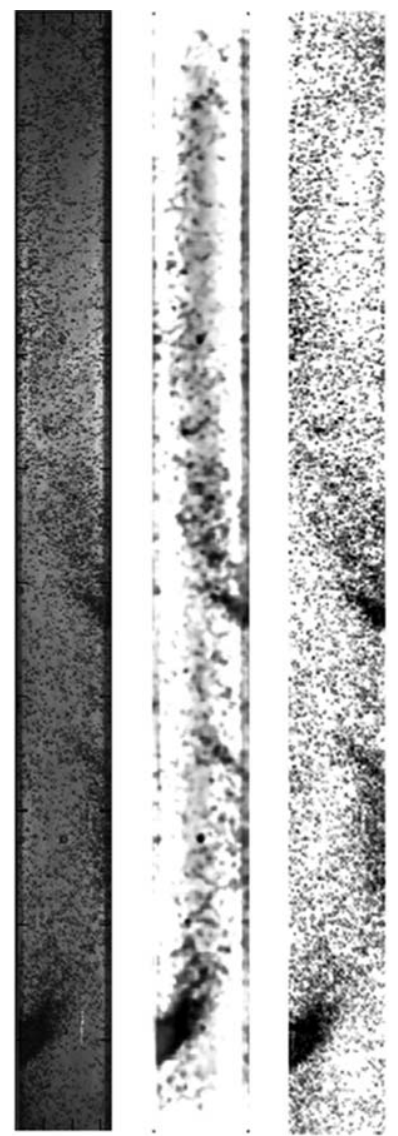

Figure 8. Post-processing of experimental images. Left: Original image. Middle: normalized image obtained from conventional DIA method. Right: normalized image obtained from THM processing.

normalized image. Even after applying image filtering, the information of the normalized image is very unsatisfactory, because the excessive use of these filters significantly distort the image information as it can be clearly noticed in Figure 7. In addition, there are spots and other stains that the original image does not contain, showing a clear contamination of the hydrodynamic information. Since image information due to shadows and background noise cannot be filtered out completely, an iterative procedure to optimize the kernel parameters is needed to minimize the influence of image imperfections. (Details of this procedure are explained in Appendix A). This makes the technique also difficult to apply well in practice.

The video recording of a fluidized bed is characterized by stationary image corresponding to the riser superimposed with the possible lighting imperfections such as uneven illumination, shadows, etc. These distortions are mostly stationary in time, while the particles are the only moving objects within the domain. In Figure 7, it can be observed that for synthetic images THM normalization method successfully removes all image imperfections, leaving the hydrodynamic information of the synthetic image unaltered. Thus, the application of a temporal image filter is a much more efficient solution when stationary distortion artifacts are aimed to be removed. Moreover, it does not require any image filter to remove spots or shadows, obtaining a normalized image of very high quality, and avoiding iterative procedures to optimize the image normalization. This is a clear evidence of the robustness of this method that normalizes the image information independently of the presence of external features.

Case 3. In this third case, we compare the performance of both DIA techniques under experimental conditions, where poor lighting is unavoidable due to the size of the illuminated area. The conventional DIA method requires image filters which are spatially applied. To optimize the image normalization by means of the conventional technique, several iterations of image filter kernel sizes have been performed. The optimal result of the conventional DIA for this case is shown in Figure 8. Further details about this procedure are found in Appendix A.

Although most of the hydrodynamic information is transferred to the normalized image in the case of conventional DIA, it can be noticed that intensity changes due to shadows close to the walls are not completely removed. In addition, the employment of big normalization kernels enhances solids volume fraction overestimation due to the fact that the intensity of the shadows is not removed.

Figure 8 also shows the normalized image produced by the THM method. It can be noticed that the intensity due to shadows, or noise, is completely removed and that only the intensity due to particles presence is transferred to the normalized image. Furthermore, the image is not as distorted as the one generated using the conventional DIA method, because THM is a method free of tuning. In other words, this method does not require the employment of image filtering techniques, whose kernel parameters need to be optimized when lighting imperfections are captured by the camera.

Thus, the conventional DIA post-processing method, which employs local image filtering techniques, significantly impedes the quality of the hydrodynamic measurements using the PIV/DIA technique. Hence, we claim that the temporally histogram-based DIA method normalizes the hydrodynamic information contained in images much more accurately than the conventional DIA method. In addition, the novel method shows a strong robustness under adverse

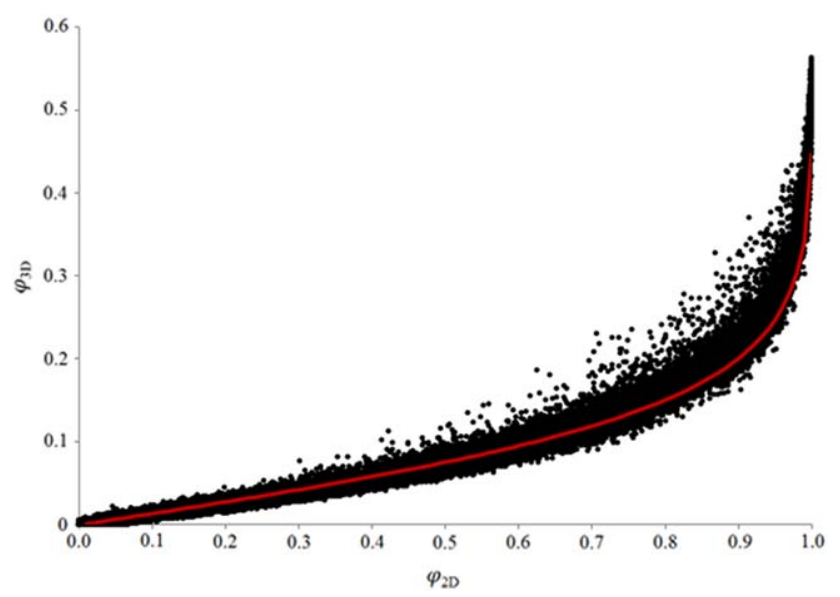

Figure 9. Normalized Intensity vs. 3D solids volume fraction from simulation data. 191,000 data points.

[Color figure can be viewed in the online issue, which is available at wileyonlinelibrary.com.] 
imaging conditions, as it can be noticed after analysis of the three cases reported in this section.

\section{$2 D$ Intensity vs. 3D solids volume fraction correlation}

In this section, a correlation to translate the 2D normalized intensity data into a solids volume fractions is developed. Moreover, a sensitivity analysis of the model is performed as well as its dependence on image-related parameters and the ratio between particle diameter and bed-depth, $d_{p} / D$.

In Figure 9, around 191,101 post-processed intensity values of synthetic images are plotted against the solids volume fraction data that have been extracted from its corresponding simulation. Here the 2D-intensity data is averaged over windows of $8 \times 8$ pixels, the corresponding 3D solids volume fractions are obtained on grid cells of the same size and averaged in the depth-direction. It is observed that the pattern follows a straight line at low and moderate intensities, while it tends to get steeper at intensities from 0.8 onwards to finally tend to infinity at intensities around 1.0. It can also be noticed that the scattering increases at higher intensity values. This is easily explained by the fact that dense regions are more susceptible to inaccuracies because particles in the front layers block the camera view and there is less visual access to estimate the solids volume fraction.

It is noted that the functional form that best correlates the normalized intensity to the $3 \mathrm{D}$ solids volume fraction for our case is given by Eq. 3, where $B$ represents the value where the asymptote is placed, while $A$ fixes the slope of the curved at low values. Equation 3 is different than correlations reported by Van Buijtenen et al. ${ }^{19}$ and De Jong et al. ${ }^{20}$. The root cause of this difference resides in the design features of the synthetic images that were employed for the 2D-3D correlation. Our synthetic images were designed to more closely resemble our experimental observation (see Figure 2). Unlike Van Buijtenen et al. ${ }^{19}$ and De Jong et al. ${ }^{20}$, in our synthetic images, the particle intensity does not change along the bed depth and subpixel intensity is implemented.

Sensitivity Analysis. To evaluate the sensitivity of the solids volume fraction correlation, we post-process different simulations under diverse imaging conditions that are specified in detail in Appendices A-D. We will show that the $A$ and $B$ parameters of the correlation mainly depend on the $d_{p} / D$ ratio, and does not need to be calibrated using, e.g., the solids weight, enabling the application of the new DIA technique in riser flows.

As stated by De Jong at el. ${ }^{20}$, the particle size-depth ratio has a direct influence on the hydrodynamic behavior of a fluidized system. This ratio must not be too high and not too low. Although high values would decrease the particle blocking effects, this would lead to a hydrodynamic behavior that is too much influenced by the walls, which clearly is an unwanted feature for this sort of studies. Conversely, ratios that are too low lead to inaccuracies in the vector field computation when PIV is employed, as well as in the solids volume fraction measurements of dense fluidized systems. Anyhow, the model parameters dependence at different $d_{p} / D$ ratios is analyzed, keeping the image resolution and contrast constant.

In experiments A-E of Table 3, the value dependency of parameters $A$ and $B$ on $d_{p} / D$ are listed, observing a noticeable dependence of constant $A$, while constant $B$ is quite stable and approximately constant. Thus, we can state that only parameter $A$ depends on the $d_{p} / D$ ratio, while $B$ could be fixed to a constant value.

In Table 3 , we present $A$ and $B$ values at different $d_{p} / D$ ratios. It can be noticed that only $A$ strongly depends on the
Table 3. Dependencies of A and B on Bed-Depth and Resolution

\begin{tabular}{cccccc}
\hline Experiment & $d_{p} / D$ & $\frac{d_{p}}{h}$ & $A$ & $B$ & $R^{2}$ \\
\hline A & 0.100 & 2.429 & 0.089 & 0.99 & 0.982 \\
B & 0.167 & 2.429 & 0.140 & 0.99 & 0.986 \\
C & 0.217 & 2.429 & 0.174 & 0.99 & 0.989 \\
D & 0.263 & 2.429 & 0.203 & 1.00 & 0.992 \\
E & 0.333 & 2.429 & 0.246 & 1.00 & 0.993 \\
F & 0.167 & 1.457 & 0.088 & 0.99 & 0.988 \\
G & 0.167 & 2.001 & 0.139 & 0.99 & 0.987 \\
H & 0.167 & 3.157 & 0.138 & 0.98 & 0.983 \\
I & 0.167 & 3.786 & 0.140 & 0.98 & 0.977 \\
\hline
\end{tabular}

$d_{p} / D$ ratio, whereas $B$ is constant always even under strong changes in the contrast and $d_{p} / h$. To apply the correct values of $A$ and $B$ on a given experiment, we have fitted their respective dependence into the equations shown below:

$$
\begin{gathered}
A\left(d_{p} / D\right)=0.6818 \cdot d_{p} / D+0.024 \pm 0.001 \\
B=0.99 \pm 0.01
\end{gathered}
$$

By means of Eqs. 4 and 5, THM can be applied in DIA experiments where the $d_{p} / h$ ratio is higher than 2 pixels per particle diameter.

As it was previously explained in the introduction, $A$ would be expected to be approximately equal to $2 d_{p} / 3 D$ for very dilute regions. Actually, at low-mid intensity values (from $\left.\varphi_{2 \mathrm{D}}=0-0.5\right)$ of the slope $A$ vs $d_{p} / D$ is very close to $2 / 3$, and it has a small offset (0.024), although.

Since the resolution of a recorded object depends on the number of pixels that defines it, the image resolution is another parameter to be included in the sensitivity analysis. In this subsection, the image resolution is quantified in pixels per particle, which is required to attain an adequate representation of the particles and consequently an accurate estimate of the solids volume fraction. To study this effect, four sets of 1200 synthetic images with different resolution were generated to study this effect. The resolution was quantified in terms of pixel per particle, $d_{p} / h$, with $h$ being the size of a pixel in metric units [meters/pixel].

In experiments B and $\mathrm{F}-\mathrm{I}$ of Table 3, we show that the value $A$ is significantly higher when very low resolution images are post-processed (1.45 pixels per particle). However the values of both constants are very stable when a ratio of at least 2 pixels per particle is used. Thus, we suggest using ratios higher than 2 pixels per particle to reach a higher stability of the constant values of the model. This value nicely matches with one of the findings reported by Westerweel ${ }^{33}$ for PIV, namely that PIV measurements are more accurate when the $d_{p} / h$ ratio is above two. Thus the model for correlating normalized intensity to solids volume fraction is also quite stable when ratios above two are employed, again highlighting the stability of the THM method against shadows and image imperfections that are unavoidably captured by the camera when large areas are recorded.

To evaluate the influence of the contrast we computed the values of $A$ and $B$ under different image contrasts. As expected, $A$ and $B$ remain unchanged for varying contrasts at the same values of $d_{p} / D$ and $d_{p} / h$. So, we can confirm that by applying THM, the contrast does not have any impact on the solids volume fraction measurements.

\section{THM accuracy}

In this subsection, the accuracy of the THM correlation is analyzed. Solids volume fractions from simulation data were 


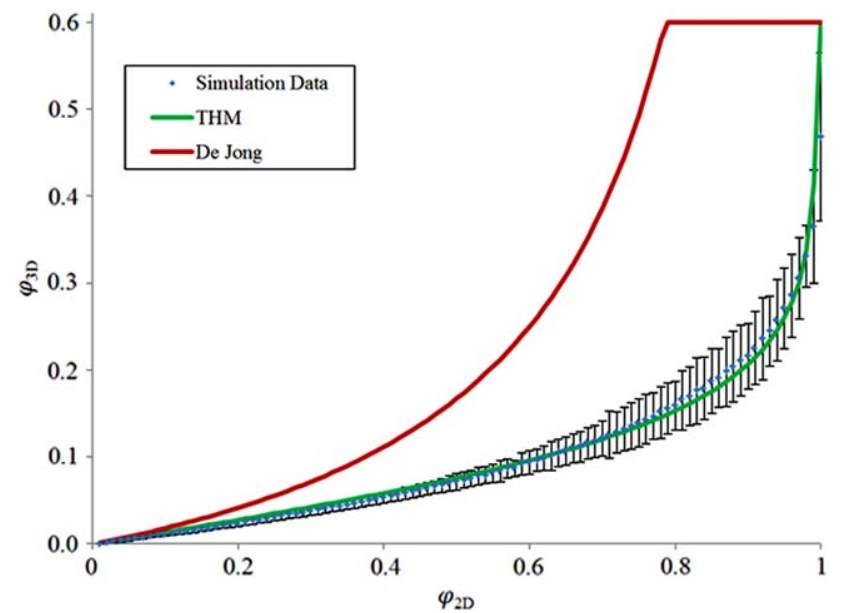

Figure 10. Solids volume fraction from simulation data vs. estimation of THM and De Jong 2D-3D correlations. ${ }^{20}$

[Color figure can be viewed in the online issue, which is available at wileyonlinelibrary.com.]

employed as a reference to compare with solids volume fraction estimations from post-processed synthetic images from the corresponding simulation. The 2D normalized intensity of the synthetic images was correlated to their corresponding solids volume fraction data from simulations. The comparison between both DIA methods have been performed using synthetic images without defects due to shadows etc., such that the $2 \mathrm{D}$ intensity can be determined also with the conventional DIA without a problem. Thus, this test solely evaluates the accuracy of the respective 2D-3D correlations over images that are already normalized and where image distortion factors are completely absent.

Previous authors ${ }^{19,20}$ proposed a correlation to translate intensity values into solids volume fraction data by using the equation given below:

$$
\left\{\begin{array}{l}
\varphi_{3 D, \max } \text { for } \varphi_{3 D} \geq \varphi_{3 D, \max } \\
\frac{A \cdot \varphi_{2 D}}{1-\varphi_{2 D} / B} \text { for } \varphi_{3 D}<\varphi_{3 D, \max }
\end{array}\right.
$$

In Figure 10, around 191,101 simulation data points of solids volume fraction were binned into 100 bins according to their $2 \mathrm{D}$ intensity. The case shown in this figure corresponds to experiment $\mathrm{B}$, which $d_{p} / h$ and the $d_{p} / D$ ratios are 2.429 and 0.167 , respectively. The bin-averaged solids volume fraction data were computed and represented by blue dots. This data set is accompanied by error bars that indicate the standard deviation $(68 \%)$ interval between the 3D solids volume fraction of each of these 191,101 simulation data points and the averaged 3D solids volume fraction, conveying the distributed nature of the data. Equations 3 and 6 have been evaluated over the $2 \mathrm{D}$ intensity range to compare the precision of their respective estimates. It is noted that values that tend to infinity or exceed the maximum packing fraction are set to $\varphi_{3 \mathrm{D} \text {, max }} \approx$ 0.6 .

According to De Jong et al. ${ }^{20}$, the values of $A$ and $B$ of Eq. 6 under these experimental conditions are $A=1.028 \cdot d_{p} / D$ $=0.171$ and $B \cong 1.005$. In case the THM is applied, the values of $\mathrm{A}$ and $\mathrm{B}$ under these conditions are 0.1397 and 0.9904, respectively. As it can be observed, there is a clear overestimation of the solids volume fraction data using the correlation of De Jong et al. (Further explained in Appendix A). In contrast, it is shown that the THM correlation is much closer to the true reference (simulation data) throughout the whole 2D intensity range, only slightly overestimating the solids volume fraction at very high $2 \mathrm{D}$ intensities. The total relative error of THM DIA method is of $15 \%$, while Eq. 6 shows a relative error of $147 \%$, revealing that the proposed $2 \mathrm{D}-3 \mathrm{D}$ correlation in this work is significantly more accurate than Eq. 6.

Clearly, in the De Jong method, the $A$ and $B$ parameters could be refitted to give a better representation of the data. The point is, however, that the literature recipes for determining $A$ and $B$ in the model of De Jong et al. do not work well in our case of riser flow. Instead of refitting $A$ and $B$ we also choose a different functional form, namely, Eq. 3 that was found to be able to fit the data better. Even for this functional form there is a systematic deviation from $\varphi_{2 D}=0.6$ onward although the curve remains well within the $68 \%$ interval. Unfortunately we were not able to find a simple functional form that was able to capture the full trend even more accurately. As an alternative to the "arctanh" of Eq. 3 one could use a spline fit but still use the same relations for $A$ and $B$ as we provided before, i.e., $\varphi_{3 D}=A \cdot B \cdot \mathrm{f}\left(\varphi_{2 D} / B\right)$, with $\mathrm{f}$ the spline function. Also note that the actual fitting was done by fitting a function of $\varphi_{2 D}$ as function of $\varphi_{3 D}$ because in the simulation $\varphi_{3 D}$ is known and $\varphi_{2 D}$ has a distribution of values. See Appendix B for an explanation of the used fitting procedure.

Thus, in this subsection we have shown that the correlation proposed in this work is more accurate than the reported by previous authors. To have a deeper insight about the accuracy of this method, we computed the solids weight of a closed system with known solids content.

The particles loaded into the riser were weighed after the experiments were performed (34.4 g). The gas superficial velocities were such that the particles never left the recorded domain (see Table 4). During the fluidization, a small amount of particles were in a stagnant region of the recycling pipe that connects the bottom of the riser. This volume of this region was estimated to compute the amount of solids that were not captured during the recording. Assuming that this stagnant volume was completely packed of particles $\left(\left\langle\varphi_{s}\right\rangle \approx 0.6\right)$, we estimate that around $3.9 \mathrm{~g}$ of particles were not captured in the recording. Thus the real amount of particles that were fluidized in the system is estimated to be $30.5 \mathrm{~g}$.

Recordings of 3,000 images under three different gas superficial velocities were post-processed. The solids volume integral of the recorded area was computed to calculate the solids weight of the system. To test the accuracy of this technique, we compared the computed value from post-processed images and the real weight amount of solids in the system (30.5 g).

As result, the time-averaged solids volume fraction field is obtained and the solids content of the system is computed from these data:

Table 4. Experiments in Closed Recording System

\begin{tabular}{lcccc}
\hline $\mathrm{U}(\mathrm{m} / \mathrm{s})$ & $\begin{array}{c}\left\langle\operatorname{mass}_{\text {Real }}\right\rangle \\
(\mathrm{g})\end{array}$ & $\begin{array}{c}\left\langle\operatorname{mass}_{\text {DIA }}\right\rangle \\
(\mathrm{g})\end{array}$ & $\begin{array}{c}\sigma\left(\operatorname{mass}_{\text {DIA }}\right) \\
(\mathrm{g})\end{array}$ & $\begin{array}{c}\text { Relative } \\
\text { error }(\%)\end{array}$ \\
\hline 3.97 & 30.5 & 30.84 & 2.20 & 3.59 \\
3.17 & 30.5 & 25.41 & 2.07 & 16.69 \\
2.38 & 30.5 & 26.13 & 1.69 & 18.33 \\
\hline
\end{tabular}


Table 5. Particle Intensity Fluctuations

\begin{tabular}{lcccc}
\hline Experiment & $f$ & $A$ & $B$ & $R^{2}$ \\
\hline B & 0 & 0.140 & 0.99 & 0.986 \\
B_01 & 0.1 & 0.146 & 0.95 & 0.986 \\
B_02 & 0.2 & 0.154 & 0.90 & 0.984 \\
B_04 & 0.4 & 0.172 & 0.81 & 0.977 \\
\hline
\end{tabular}

$$
\left\langle\operatorname{mass}_{\text {DIA }}\right\rangle=\sum_{i=1, j=1}^{N}\left\langle\varphi_{s}\right\rangle_{i, j} \Delta x \Delta y D \rho_{s}
$$

where, $\rho_{\mathrm{s}}$ is the solids density; $\left\langle\varphi_{\mathrm{s}}\right\rangle_{\mathrm{i}, \mathrm{j}}$ is the time-averaged solids volume fraction of an interrogation area; $\mathrm{N}$ is the total number of interrogation areas in the image; $\Delta x$, and $\Delta y$ are the dimensions of an interrogation area; and $D$ is the depth of the riser.

These results show that the error for time-averaged results of solids volume fraction measurements, is lower than $20 \%$.

Influence of Temporal Fluctuations. The necessary assumption for the THM to work so well is that there is a oneto-one correspondence between the coverage of a pixel by a passing particle and the measured intensity. In practice, this one-to-one correspondence might not be exactly obeyed. Reasons for this might be: variations in particle color or tone, accidental reflections to irregular particle surfaces, camera noise, fluctuations in the light intensity, etc.

As a consequence, maximum of the histogram might exceed the average "uncovered pixel" value, while the minimum will be less than the average "fully covered pixel" value. Therefore, when using the width of the histogram for normalization, the value for $\varphi_{2 D}$ that is computed for a fully uncovered pixel will be typically larger than 0 , and the value computed for a fully covered pixel typically less than 1 (instead of exactly equal to 0 and 1 ).

To illustrate this phenomenon, we have generated four sets of synthetic images where the particle intensity varies according to the equation below:

$$
\begin{aligned}
& I_{\text {particle }}(i, j)=((1-f)+f \cdot \text { unif }()) \\
& \quad \cdot\left(I_{0}(i, j)-I_{\text {background }}(i, j)\right)+I_{\text {background }}(i, j)
\end{aligned}
$$

where $I_{0}(i, j)$ is the core intensity of the darkest particle that has passed by a single pixel, $I_{\text {background }}(i, j)$ is the background intensity and $f$ is the randomness factor that defines the magnitude of the intensity fluctuation of the particle at that pixel position. This would correspond to a case where particles have different colors or tones and therefore the intensity of the reflected light differs for each particle. For instance, a $f$ value of 0.5 would mean that the particle intensity fluctuates between the temporal minimum intensity of a pixel and the middle intensity of the temporal histogram (see Figure 1).

Using simulations and "measuring" the $\varphi_{3 D}-\varphi_{2 D}$ pairs for the different $8 \times 8$ pixel sized-windows we have evaluated the influence of $f$ on the fitting parameters $A$ and $B$, with the values listed in Table 4.

As we can see in Table 5, particle color inhomogeneity has a noticeable effect on the fitting parameters, leading to higher slopes and lower $B$ values to overcome an underestimation of the solids volume fraction. The message of this result is that in experiments, one should as much as possible avoid this type of temporal fluctuations. For example, make sure that the color differences between particles is minimal and minimize the influence of camera noise and fluctuations in the lighting. If fluctuations are still present, one might try to quantify those, e.g., by a parameter $f$ and its influence on the values of $A$ and
$B$. The quantification of $f$ in experiments is further explained in Appendix D.

\section{Application of THM on Riser Flow: Solids Volume Fraction Measurements}

In this section as a "proof of principle," we show some representative results obtained from a single experiment by applying the new DIA method in combination with PIV. It has been shown that the THM DIA technique enables full-field accurate measurement of the solids volume fraction under riser flow conditions. The temporal character of the histogram-based normalization enables the experimental determination of riser axial profiles without concern about the illumination conditions. In this section, we show some representative results obtained from a single experiment by applying the new DIA method in combination with PIV. ${ }^{12}$

This experiment was performed in the pseudo-2D riser, of which the dimensions are described in section 2 . In this case, black $0.8-0.9 \mathrm{~mm}$ glass beads $\left(\rho_{\mathrm{s}}=2500 \mathrm{~kg} / \mathrm{m}^{3}\right)$ were fluidized with air at room temperature and atmospheric pressure. The gas superficial velocity was of $U=5.95 \mathrm{~m} / \mathrm{s}$ and the solids mass flux of $G_{s} \cong 31 \mathrm{~kg} / \mathrm{m}^{2} \mathrm{~s}$. According to the characteristics of the particles that were employed on this experiment, $A$

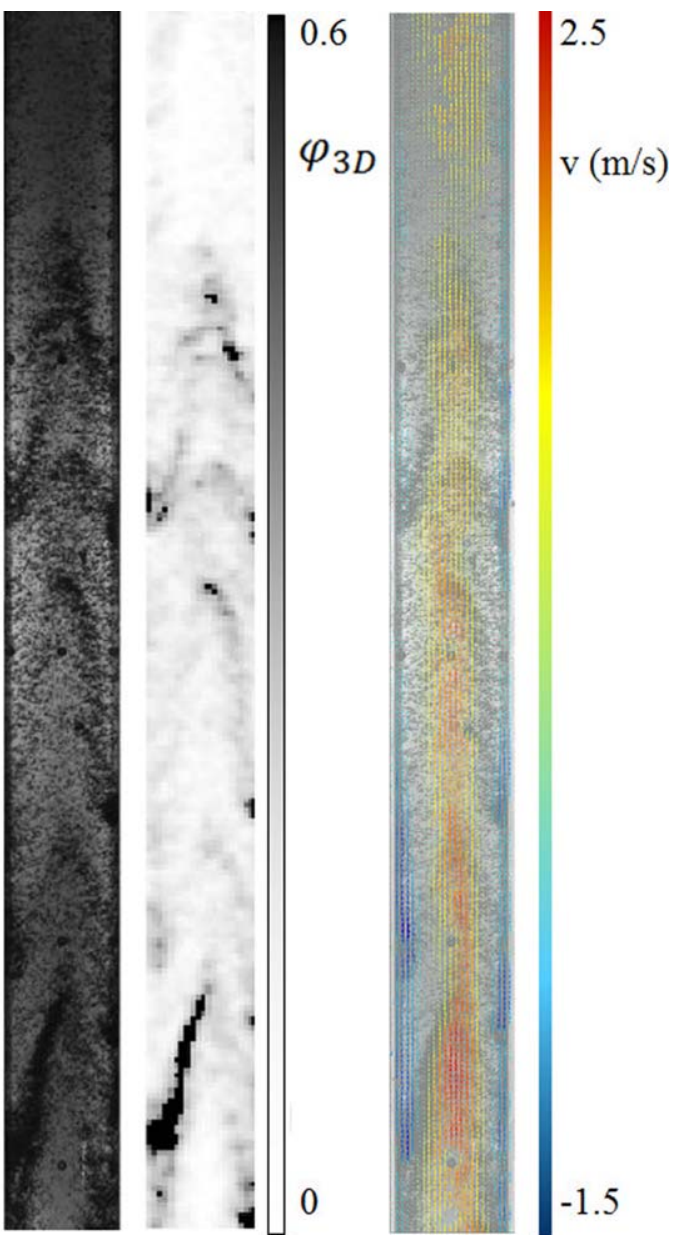

Figure 11. Instantaneous solids volume fraction and solids velocity field of an instantaneous image of the top section of an experimental pseudo-2D riser.

[Color figure can be viewed in the online issue, which is available at wileyonlinelibrary.com.] 


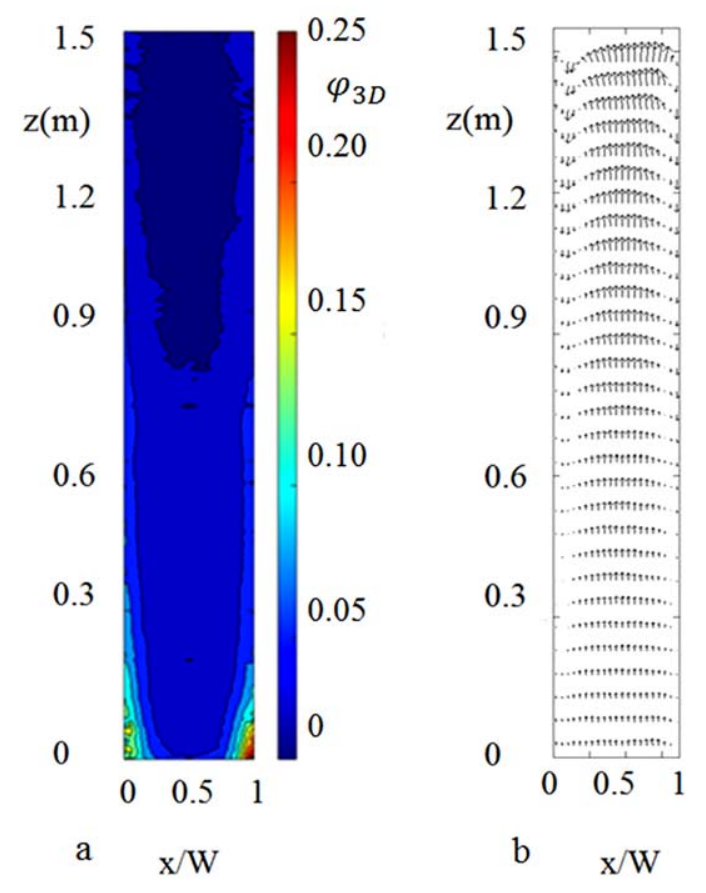

Figure 12. (a) Full-field time-averaged solids volume fraction. (b) time-averaged solids mass flux vector over full flow field.

[Color figure can be viewed in the online issue, which is available at wileyonlinelibrary.com.]

and $B$ values, respectively, were 0.144 and $0.86\left(d_{p} / D=0.142\right.$ and $f=0.3$ ), respectively, as result of using equations reported in Appendix D.

By means of an image cross correlation between two consecutive images, PIV supplies an individual solids velocity vector for each interrogation area of $8 \times 8$ pixels:

$$
\bar{v}_{p}=\frac{\bar{s}_{p}(\bar{x}, t)}{M \cdot \Delta t}
$$

where $M$, is the magnification of the camera, $\bar{s}_{p}$, represents the solids displacement vector in a time interval of $\Delta t$, which would be the time delay between two images. However, the application of PIV only provides a solids velocity vector field, which is not sufficient to describe the solids motion of the system. In heterogeneous systems, both solids volume fraction and solids mass flux data are necessary parameters to describe their hydrodynamics. Thus, the measurement of accurate solids volume fraction data is essential to characterize the hydrodynamic behavior of a riser in more detail.

In Figure 11, the instantaneous solids volume fraction and solids velocity fields of an experimental image are shown, which can be used to determine hydrodynamic information of the whole recording field. All the image imperfections are successfully removed and only the presence of particles is accounted for, providing a data set covering a domain size of $0.07 \times 0.8 \mathrm{~m}$, subdivided into $21 \times 240$ interrogation areas. The application of this technique enables a full characterization of riser hydrodynamics. To obtain hydrodynamics of the full riser, two sets of experiments for the bottom and top sections were performed, covering a total riser height of $1.5 \mathrm{~m}$.

The combination of PIV and the novel DIA post-processing method supplies whole field solids volume fraction and solids mass flux data. It therefore enables the computation of the time-averaged solids mass flux for each interrogation area as:

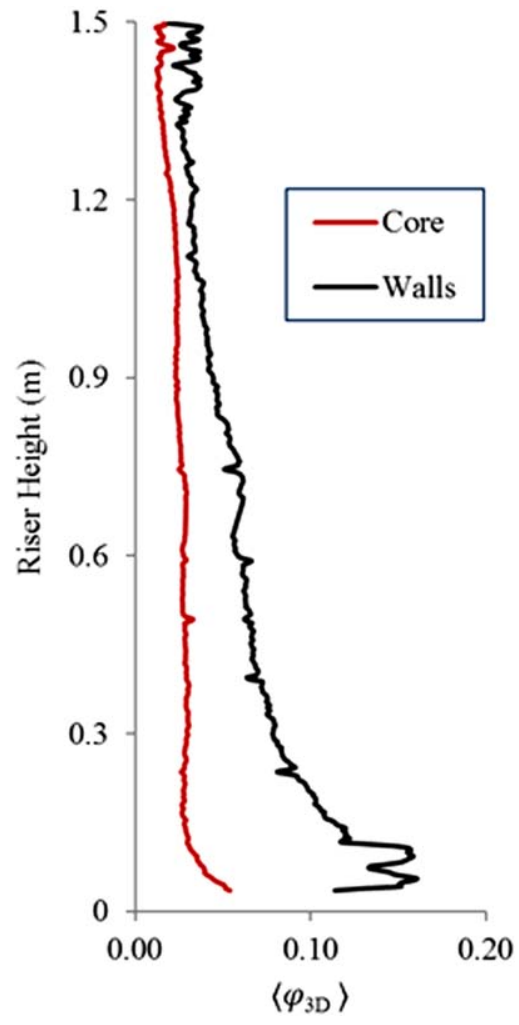

Figure 13. Riser axial profile of solids volume fraction.

[Color figure can be viewed in the online issue, which is available at wileyonlinelibrary.com.]

$$
\left\langle\bar{G}_{s}\right\rangle=\rho_{s}\left\langle\varphi_{3 D} \cdot \bar{v}_{p}\right\rangle
$$

Figure 12 shows the 2D field data sets of the time-averaged solids volume fraction and solids mass flux. These results illustrate that this technique enables experimental measurement of over the whole flow field.

To provide a more quantitative reference, we provide axial and cross-sectional profiles of these two parameters. In Figure 13, the time-averaged axial solids volume fraction profile is shown in the core of the riser and close to the wall

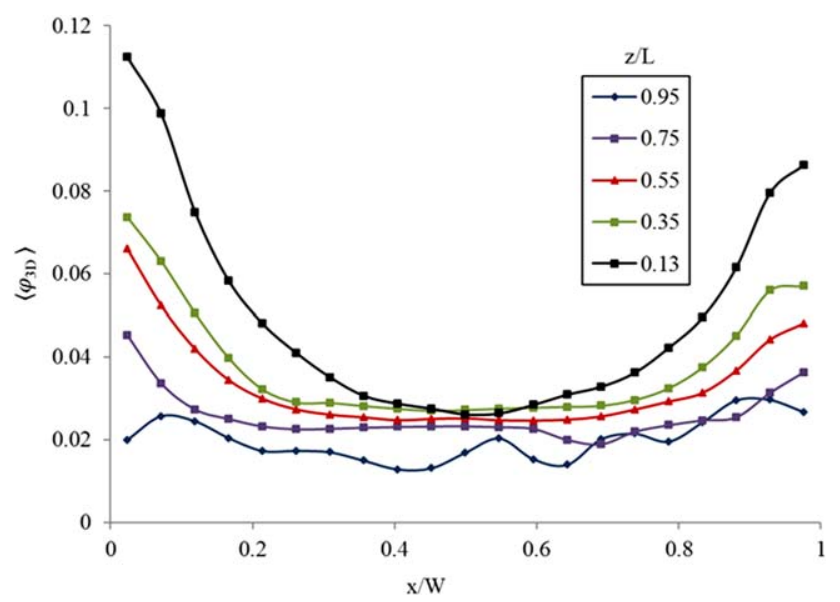

Figure 14. Time-averaged lateral profiles of the solids volume fraction at several dimensionless heights in the riser.

[Color figure can be viewed in the online issue, which is available at wileyonlinelibrary.com.] 


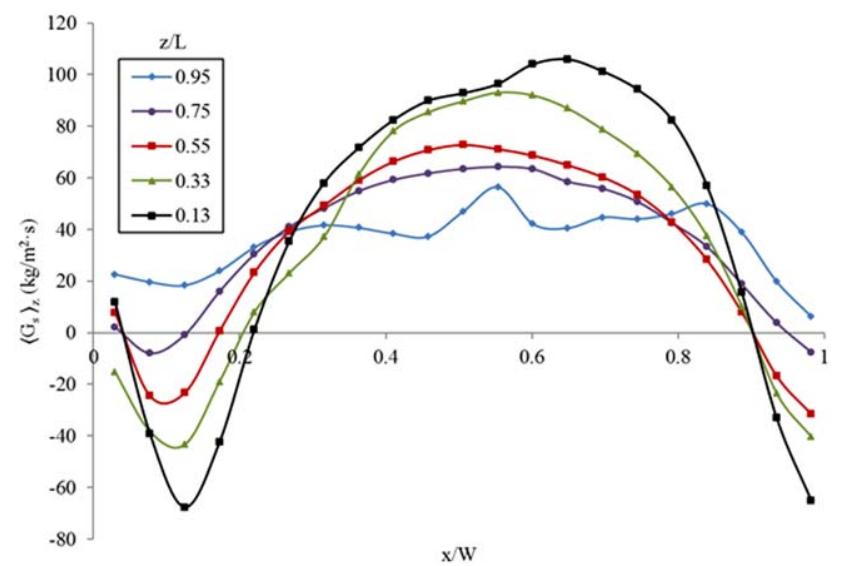

Figure 15. Time-averaged cross-sectional solids mass flux profiles at different height of the pseudo-2D riser.

[Color figure can be viewed in the online issue, which is available at wileyonlinelibrary.com.]

(showing measured values at all interrogation areas which centered $\mathrm{x}$-coordinate are $\mathrm{x}=0.0017 \mathrm{~m}$ and $\mathrm{x}=0.032 \mathrm{~m}$, respectively). The solids volume fraction decreases along the axial direction. It can be seen that there are some fluctuations at the top and the bottom of the "wall" solids fraction profile. This is due to the camera noise fluctuations, which are more significant in the least illuminated areas, which are the top and bottom areas that are close to the walls.

In Figure 14, time-averaged cross-sectional profiles of the solids fraction at different heights are shown, where U-shaped profiles of the solids volume fraction are observed, describing well the core-annulus pattern under riser flow conditions.

In Figure 15, it is observed the cross-sectional profiles of solids mass flux at different heights of the riser using PIVTHM DIA method. The profiles show an asymmetry between left and right, most probably due to the lateral solids inlet at the bottom left side of the riser.

It has to be noted that the aim of showing these results here is to illustrate the potential of THM method to provide accurate hydrodynamic results over the whole recorded field, without getting much into discussion about the influence of operational conditions on riser flow.

\section{Conclusions}

In this work, a temporal histogram-based DIA processing is presented. Its novelty resides in the temporal normalization of the processed images, whose imperfections due to uneven lighting and shadows are completely removed without the need of spatial image filtering techniques. THM provides a high-quality image normalization procedure under adverse visualization conditions, enabling the employment of the DIA technique to quantify solids volume fraction data on lab-scale pseudo-2D fluidized beds. Moreover, a new 2D-3D correlation is developed through the calibration of post-processed synthetic images and CFD-DEM solids volume fraction data. This correlation relates the intensity of the normalized images to the 3D solids volume fraction of a riser, which solids content dynamically evolves and hence is unknown at any instant.

This correlation adopts the form given by Eq. 3, which constants $(A$ and $B)$ can be fixed at the beginning of the experiment. It has been also shown that these constants depend on image-related properties such as image bed-depth and resolution; confirming that ratios higher than 2 pixels per particle are needed to accurately quantify the solids volume fraction data. The performed sensitivity analysis, shows that THM is more accurate than current full-field DIA techniques and enables its applicability to pseudo-2D risers, without need of any further calibration with the solids weight of the system.

The novel THM DIA method that has been developed increases the accuracy of solids volume fraction measurements, enabling a more thorough and complete hydrodynamic study with the PIV/DIA technique. This technique can supply generous amounts of experimental references for CFD models validation procedures.

\section{Acknowledgment}

This research is funded by The Netherlands Organization for Scientific Research (NWO) under project number 713.012.002.

\section{Notation}

\section{Roman Symbols}

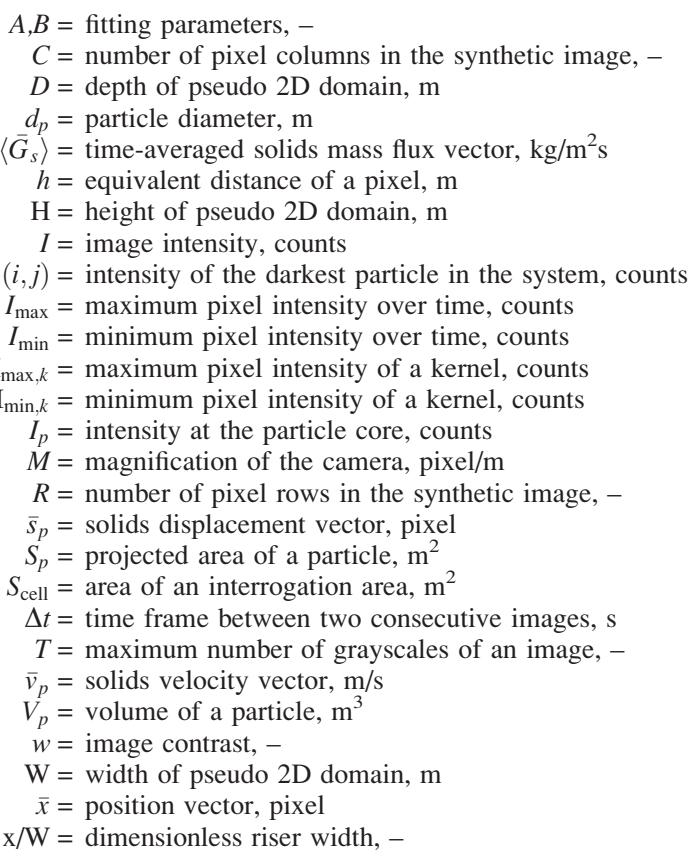

\section{Greek Symbols}

$\varphi_{3 \mathrm{D}}=$ solids volume fraction, $\mathrm{m}^{3}$ solid $/ \mathrm{m}^{3}$ riser $\varphi_{2 \mathrm{D}}=$ normalized $2 \mathrm{D}$ intensity, dimensionless $\rho_{s}=$ solids density, $\mathrm{kg} / \mathrm{m}^{3}$

\section{Literature Cited}

1. Kunii D, Levenspiel O. Fluidization Engineering. ButterworthHeinemann Series in Chemical Engineering. Newton, Massachussets, USA: Butterworth-Heinemann Limited, 1991.

2. Werther J. Scale-up modeling for fluidized bed reactors. Chem Eng Sci. 1992;47(9):2457-2462. doi:10.1016/0009-2509(92)87076-3.

3. Grace JR. High-velocity fluidized bed reactors. Chem Eng Sci.1990; 45(8):1953-1966. doi:10.1016/0009-2509(90)80070-u.

4. Singh B, Fryer C, Potter OE. Solids motion caused by a bubble in a fluidized bed. Powder Technol. 1972;6:239-44. doi:10.1016/00325910(72)83018-3.

5. Patience GS, Chaouki J. Gas phase hydrodynamics in the riser of a circulating fluidized bed. Chem Eng Sci. 1993;48(18):3195-3205. doi:10.1016/0009-2509(93)80205-5.

6. Cocco R, Cleveland J, Harner R, Chrisman R. Simultaneous in-situ determination of particle loadings and velocities in a gaseous medium. AIChE Symp Ser. 1995;91(308):147-153. 
7. Liu J, Grace JR, Bi X. Novel multifunctional optical-fiber probe: I. Development and validation. AIChE J. 2003:49(6):1405-1420. doi: 10.1002/aic.690490607

8. Tartan M, Gidaspow M. Measurement of granular temperature and stresses in risers. AIChE J. 2004;50(8):1760-1775. doi:10.1002/ aic. 10192 .

9. McMillan J, Shaffer F, Gopalan B, Chew JW, Hrenya C, Hays R, Karri SBR, Cocco R. Particle cluster dynamics during fluidization. Chem Eng Sci. 2013;100:39-51. doi:10.1016/j.ces.2013.02.047.

10. Zhang W, Wang C, Yang W, Wang CH. Application of electrical capacitance tomography in particulate process measurement-a review. Adv Powder Technol. 2014;25(1):174-188. doi:10.1016/ j.apt.2013.12.003.

11. Mathiesen V, Solberg T, Hjertager BH. An experimental and computational study of multiphase flow behavior in a circulating fluidized bed. Int J Multiphase Flow. 2000;26:387-419. doi:10.1016/S03019322(99)00027-0.

12. Ibsen $\mathrm{CH}$, Helland E, Hjertager BH, Solberg T, Tadrist L, Occelli R. Comparison of multifluid and discrete particle modelling in numerical predictions of gas particle flow in circulating fluidised beds. Powder Technol. 2004;149:29-41. doi:10.1016/j.powtec. 2004.09.042.

13. Müller CR, Holland DJ, Sederman AJ, Mantle MD, Gladden LF, Davidson JF. Magnetic resonance imaging of fluidized beds. Powder Technol. 2008;183:53-62. doi:10.1016/j.powtec.2007.11.029.

14. Mohs G, Gryczka O, Heinrich S, Mörl L. Magnetic monitoring of a single particle in a prismatic spouted bed. Chem Eng Sci. 2009; 64(23):4811-4825. doi:10.1016/j.ces.2009.08.025.

15. Buist KA, Van der Gaag AC, Deen NG, Kuipers JAM. Improved magnetic particle tracking technique in dense gas fluidized beds. AIChE J. 2014;60(9):3133-3142. doi:10.1002/aic.14512.

16. Bokkers GA, Van Sint Annaland M, Kuipers JAM. Mixing and segregation in a bidisperse gas-solid fluidised bed: a numerical and experimental study. Powder Technol. 2004;140:176-186. doi: 10.1016/j.powtec.2004.01.018.

17. Link JM, Cuypers LA, Deen NG, Kuipers JAM. Flow regimes in a spout-fluid bed: a combined experimental and simulation study. Chem Eng Sci. 2005;60:3425-3442. doi:10.1016/j.ces.2005.01.027.

18. Laverman JA, Roghair I, Van Sint Annaland M, Kuipers JAM. Investigation into the hydrodynamics of gas-solid fluidized beds using particle image velocimetry coupled with digital image analysis. Canad J Chem Eng. 2008; 86(3):523-535. doi:10.1002/cjce.20054.

19. Van Buijtenen MS, Börner M, Deen NG, Heinrich S, Antonyuk S, Kuipers JAM. An experimental study of the effect of collision properties on spout fluidized bed dynamics. Powder Technol. 2011; 206(1-2):139-148. doi:10.1016/j.powtec.2010.07.009.

20. De Jong JF, Odu SO, Van Buijtenen MS, Deen NG, Van Sint Annaland M, Kuipers JAM. Development and validation of a novel digital image analysis method for fluidized bed particle image velocimetry. Powder Technol. 2012;230:193-202. doi:10.1016/ j.powtec.2012.07.029.

21. Casleton DK, Shadle LJ, Ross AA. Measuring the voidage of a CFB through image analysis. Powder Technol. 2010;203(1):12-22. doi: 10.1016/j.powtec.2010.03.033.

22. Yang J, Zhu J. An alternative method to quantify solids phase separation in a rectangular CFB Riser. Powder Technol. 2015;273:76-82. doi:10.1002/cjce.22059.

23. Nimeroff JAB, Dorsey JA, Rushmeier HB. Implementation and analysis of an image-based global illumination framework for animated environments. IEEE Trans Visual Comp Graphics.1996;2(4):283298.

24. Chen RC, Fan LS. Particle image velocimetry for characterizing the flow structure in three-dimensional gas-liquid-solid fluidized beds. Chem Eng Sci. 1992;47:3615-3622. doi:10.1016/0009-2509(92)85077-O.

25. Wei X, Sheng H, Tian W. 2006. Characterizing particle dispersion by image analysis in ICFB. Int J Heat Mass Transfer. 2006;49: 3338-3342. doi:10.1016/j.ijheatmasstransfer.2006.03.013.

26. Katkovnik V, Egiazarian K, Astola J. Application of the ICI principle to window size adaptive median filtering. Signal Process. 2003; 83:251-257. doi:10.1016/S0165-1684(02)00387-0.

27. Vijaykumar VR, Santhana Mari G, Ebenezer D. Fast switching based median-mean filter for high density salt and pepper noise removal. AEU Int J Electron Commun. 2014;68(12):1145-1155.

28. Choy SSO, Chan YH, Siu WC. Adaptive image noise filtering using transform domain local statistics. Optical Eng. 1998;37(8):2290 2296. doi:10.1117/1.601750.
29. Van Der Hoef MA, Beetstra R, Kuipers JAM. Lattice-Boltzmann simulations of low-Reynolds-Number flow past mono- and bidisperse arrays of spheres: results for the permeability and drag force. J Fluid Mech. 2005;528:233-254. doi:10.1017/S0022112004003295.

30. Hoomans BPB, Kuipers JAM, Briels WJ, Van Swaaij WPM. Discrete particle simulation of bubble and slug formation in a twodimensional gas-fluidised bed: a hard-sphere approach. Chem Eng Sci. 1996;51(1):99-118. doi:10.1016/0009-2509(95)00271-5.

31. Leler WJ. Human vision, anti-aliasing, and the cheap 4000 line display. SIGGRAPH'80 Proceedings of the 7th annual conference on Computer graphics and interactive techniques. 1980;308-313. doi: $10.1145 / 800250.807509$.

32. Godse AP, Godse DA. Computer Graphics. New York, USA: Technical Publications Pune ${ }^{\mathrm{TM}}, 2009$.

33. Westerweel J. Fundamentals of digital particle image velocimetry. Measure Sci Technol. 1997;8:1379-1392. doi:10.1088/0957-0233/8/ $12 / 002$.

\section{Appendix A: Conventional DIA Method Normalization}

The conventional DIA post-processing method has been previously applied to quantify solids volume fraction for pseudo2D dense granular systems. This method usually requires local image filtering techniques, which will be explained in detail in this section.

Given a fluidized system with dark particles and light background, low intensities will represent the solid phase, and high ones the background phase. For instance, if there is a dark stain in the background, this detail will be captured on an image. This dark stain will consist of a group of pixels with variable grayscales that will be lower than the background intensity. These image intensities are post-processed and quantified as particle phase, whereas it is only an "imperfection" of the background. The objective of employing image filtering techniques is to "clean" the image of such unwanted features that could lead to inaccuracies in the DIA experiment post-processing.

Large areas are more difficult to illuminate and therefore shadows and light gradients become more prominent in the recorded images. This is where the removal of these image distortion factors becomes essential to obtain an accurate estimate of the solids volume fraction in a fluidized system. A median filter is employed to remove background noise, or what is called "salt and pepper noise, ${ }^{27}$ which consists of spots of very small size. Actually, our experimental background has many such small spots due to its manufacturing process. These spots can be easily removed by applying a median filter. The median filter fills up every pixel of the image by the median element of its neighborhood, removing low and high intensity peaks within a certain area, which is defined by the kernel. The size of the used kernel can be tuned, and therefore an increased size is used if the unwanted spots or stains have larger areas. However, in a fluidized system, particles could be removed if the kernel size clearly exceeds the particle size, losing relevant information for the DIA experiment.

In a riser, not only spots are present, but also shadows close to walls and light gradients, the areas of which clearly exceed the particle size. Therefore, the use of a median filter only is not sufficient to remove or minimize the impact of these shadows on the DIA post-processing.

Thus, apart from the median filter, a local intensity normalization is typically required. Once the median filter has been applied, the resulting intensity of each pixel can be normalized over the minimum and maximum intensities of its corresponding neighborhood, whose size is predefined by the used kernels. The aim of this local image normalization is to smooth intensities corresponding to stains of bigger size that cannot be removed 


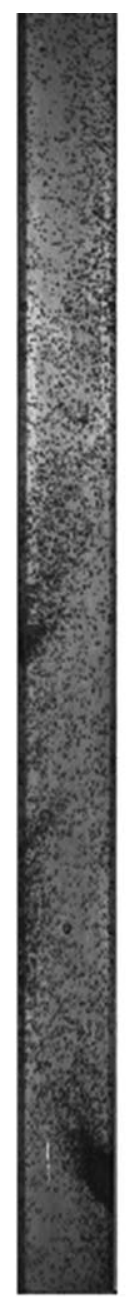

a

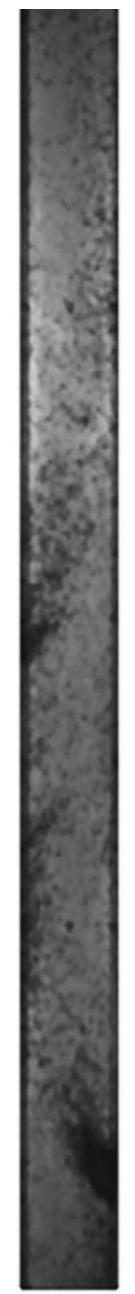

b

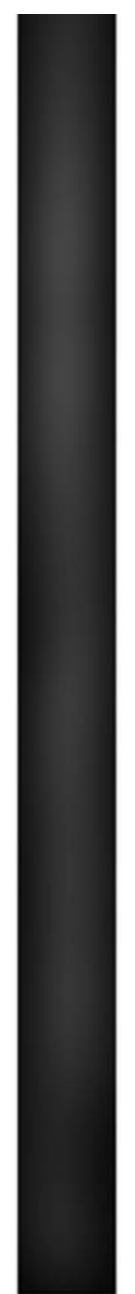

c

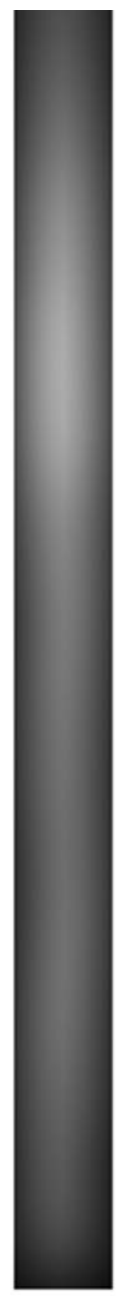

$\mathrm{d}$

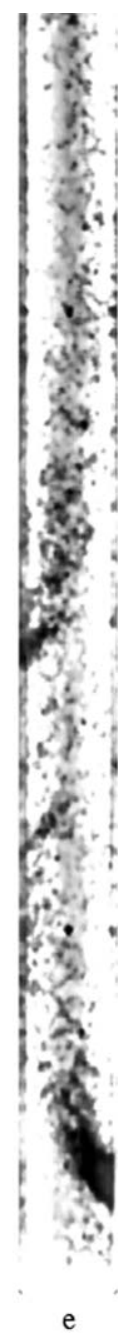

e
Figure A1. Conventional DIA Post-processing of an experimental image.

(a) Original image. (b) Median filter. (c) Blurred Minimum filter. (d) Blurred Maximum filter. (e) Normalized image.

by the median filter. Furthermore, minimum and maximum filters could be employed with a blurring filter to further attenuate the intensity of these big shadows. The normalized intensity of each pixel is expressed in Eq. A1:

$$
\varphi_{2 D}(I)=\frac{I_{\max , k}-I}{I_{\max , k}-I_{\min , k}}
$$

where $I_{\min , k}$ and $I_{\max , k}$, are, respectively, the blurred minimum and maximum intensities of the pixel neighborhood, which size is defined by the kernel employed in the image filters.

For poorly illuminated objects, this method requires a trialerror iteration, to optimize the kernel size for each of the different applied image filters. By changing the kernel size of a filter, a different outcome will eventually be obtained. This is why an iterative procedure is needed to optimize the quality of the normalized image. After several iterations, optimal kernel sizes can be found to search for the image that more closely resembles to the original one while unwanted intensities have been successfully removed (shadows, light gradients, and other image imperfections).

Figure A1 shows a sequence of the intermediate steps that are conducted to normalize image A1a. After testing different kernel sizes in an attempt to remove as many undesired image details as possible, only the optimal result is shown in image Ale.

Image A1b would be the result after application of a median filter to the original image (A1a), whereas images A1c, d are, respectively, the results of blurred minimum and maximum filters, over which the local normalization is done. Figure Ale is the final result after applying all corresponding filters. It can be seen that the particles are blurred and shadows are still present in the image, leading to significant inaccuracies in the solids volume fraction estimation.

To better illustrate the iterative nature of this procedure, a short sequence of normalized images is shown in Figure A2. In this case, a synthetic image is post-processed. Figure A2b corresponds to a synthetic image with added image imperfections such as shadows, light gradients and spotted background; whereas Figure A2a corresponds to the same image where only particles and clear background are shown, and this would represent our reference image to evaluate the quality of the image normalization.

Figure A2c shows the first normalized image after applying a median filter with a $3 \times 3$ kernel to Figure A2b. It can be observed that the outcome of the image normalization is not

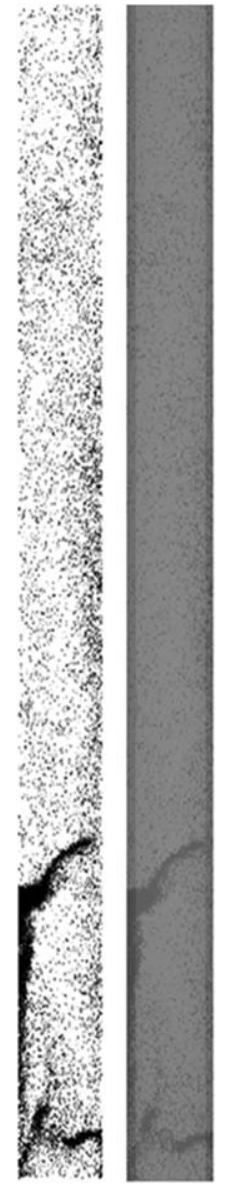

a

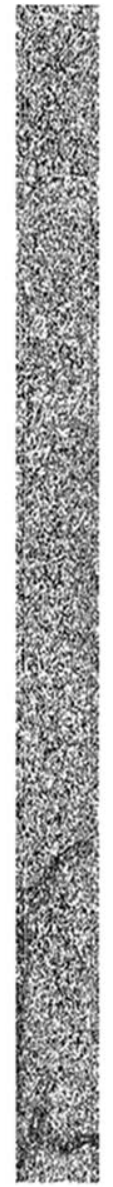

c

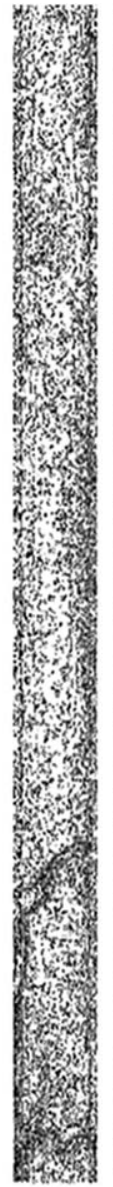

d

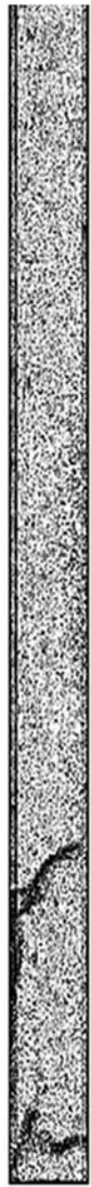

e

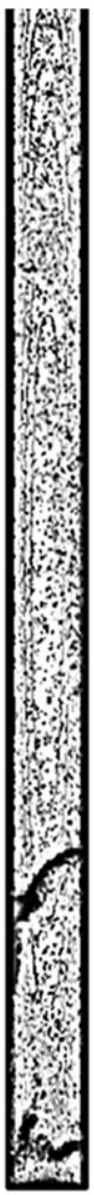

$f$
Figure A2. (a) Original image. (b) original image with shadows and other imperfections. (c) median filter $3 \times 3$ kernel. (d) $3 \times 3$ median filter plus $3 \times 3$ blurring plus normalization over $3 \times 3$ minimum and maximum filters. (e) $7 \times 7$ median filter. (f) $7 \times 7$ median filter plus $7 \times 7$ blurring plus normalization over $7 \times 7$ minimum and maximum filters. 
satisfactory, since the particles are not distinguishable from the spotted background and this would lead to large errors in the solids volume fraction quantification. Thus, we perform a second trial by increasing the kernel size of the median filter, thus obtaining image A2e. The usage of a bigger kernel further clears the image, easing the distinction between particle and background phases. However, the background still differs from image A2a, which would be our target image to reproduce.

A third trial is performed to normalize image A2b, by adding the local normalization filter given by Eq. A1 with filter kernel sizes of $3 \times 3$ pixels, gives Figure A2d. This results in an even less spotted background, but it is also noticeable as a slight blurring in the image and it is still quite hard to distinguish particle and background phases. On a final iteration, blurring, median and local normalization filters of $7 \times 7$ pixels kernels are applied giving Figure A2f. Although, Figure A1e reveals a qualitative improvement compared to the images in Figure A2, shadows close to the walls are not removed and stains are found throughout the whole image.

The usage of bigger kernels results in the loss of image details that are smaller than the size of the employed kernels (in our case, the particles, which particle diameter could be ranged between 2 and 4 pixels) and therefore alter the hydrodynamic information of the image. Thus, we limit our iteration to kernels smaller than $7 \times 7$ pixels.

Additional iterations could be performed by changing the kernel size of the utilized image filters. However, these are locally applied and therefore do not successfully isolate the desired information when poor lighting conditions are given, making this procedure tedious and time-consuming without achieving satisfactory results.

\section{Correlation 2D-3D solids volume fraction}

Synthetic images of a bubbling fluidized bed were employed to obtain Eq. 6. According to Van Buijtenen et al, the values of constants $A$ and $B$ can be obtained through an iterative procedure that minimizes the difference between the estimated solids weight (by DIA) and the real solids weight of the system. ${ }^{19}$ This method is especially suited for bubbling fluidized beds, which domain is closed and its solids content is always known and constant. Thus, this method would not be applicable for riser flow conditions.

De Jong et al. showed that the $d_{p} / D$ ratio has a noticeable influence on the 2D-3D correlation, ${ }^{20}$ which constants can be known without need of any calibration with the total solids weight contained in the bed. Constant $A$ is claimed to be dependent on a light intensity gradient that changes at different $d_{p} / D$ ratios and can be computed with the equation given below:

$$
A \approx \frac{\varphi_{3 D}}{\varphi_{2 D}} \approx \frac{V_{p} / V_{\text {cell }}}{S_{p} / S_{\text {cell }} I_{p} / I_{\max }} \approx 1.028 \frac{d_{p}}{D}
$$

De Jong et al. also reported different $B$ constant values that were claimed to be fixed at known $d_{p} / D$ ratios. ${ }^{20}$

\section{Appendix B: Fitting 2D-3D Correlation}

For each DIA experiment, 191,000 normalized intensity data points were obtained from post-processed synthetic images and correlated to the real solids volume fraction data from CFD-DEM simulations.

Each experiment was fitted to Eq. 3 by using weighted least squares method, of which the results are shown in Figure B1.

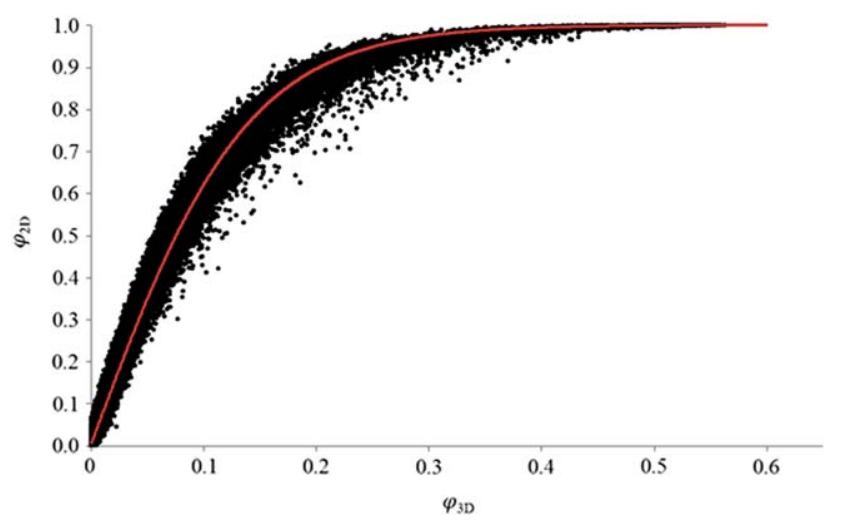

Figure B1. Experiment fitting sample: B.

[Color figure can be viewed in the online issue, which is available at wileyonlinelibrary.com.]

All these points were weighted on region basis to avoid a biased fitting due to regions with higher density of data points.

Thus, the whole solids volume fraction range was divided into 100 bins, where each point weight was inversely proportional to the number of data points of its corresponding bin. Thus, each data point weight was computed as follows:

$$
w_{i, b i n}=\frac{\sum_{i=1}^{i=N} n_{i, b i n}}{n_{i, b i n}}
$$

In this case the values the fitted values of A and B are 0.1397 and 0.9904 , respectively, with a regression coefficient equal $\left(\mathrm{R}^{2}\right)$ to 0.9861 .

\section{Appendix C: Synthetic Images}

Subpixel intensity was implemented in a homemade script to generate 8-bit synthetic images. Each pixel was subdivided into $8 \times 8$ subpixels areas to approximately quantify the fraction of particle area that was occupying the evaluated pixel.

Thus, the particle intensity was calculated as follows:

$$
I_{i, j}=\left(\frac{N_{\text {occupied }}}{64}\right) \cdot\left(I_{p}\right)+I_{\text {background }}
$$

where $\mathrm{N}_{\text {occupied }}$ is the number of subpixels that are occupied by particle phase, $I_{\text {background }}$ is the background intensity at the particle location and $I_{p}$ is the intensity of a fully occupied pixel by particle phase.

Spotted background and uneven lighting effects were empirically generated by means of the next equations:

$$
I_{i, j}=100-\operatorname{round}(8 \cdot \operatorname{unif}())+0.001 \cdot\left({ }^{C} / 2-i\right)^{2}+0.000005 \cdot\left({ }^{R} / 2-j\right)^{2}
$$

where $\mathrm{C}$ and $\mathrm{R}$, are, respectively, the number of pixel columns and rows of the synthetic image, and unif() generates uniformly distributed numbers between 0 and 1 . The intensity was further increased by 15 units to mimic shadows close to the walls of all pixels which of the $\mathrm{i}$ coordinate was lower than ceil $(0.1 \times \mathrm{L})$ and higher than ceil $(0.9 \times \mathrm{L})$.

For comparison purposes between simulation and post-processed data, images of eightfold the number of grids employed on their respective simulation were generated. For example, for experiment B, images of $2164 \times 168$ pixels were generated. Pixel-data of these images were locally averaged into $273 \times 21$ grid data fields. Thus, each image was windowed into $8 \times 8$ pixel grids to 
have the same mapping as in the simulations to enable a quantitative comparison between post-processed and computational solids volume fraction data. All sets of synthetic images had these dimensions, except those belonging to experiment I consisting of $3408 \times 264$ pixels.

\section{Appendix D: Determination of $\boldsymbol{f}$ in Experiments}

The quantification of $f$ in experiments can be performed by visual analysis. In Figure D1, we show an image sequence to determine the value of $f$. Given a normalized image, fully covered pixels should have a $\varphi_{2 \mathrm{D}}=1$. However, minima intensities that have been registered in a first processing pass could correspond to intensity values of the darkest particles of the solids inventory. If we only output those pixels which are $\varphi_{2 \mathrm{D}}=1$, all fully covered pixels by particles should be visualized. However, this could not be the case if there are fully covered pixels by lighter particles, eventually due to color inhomogeneity of the particles pigment. Thus, we have performed additional calibrations for cases where the particles of the system had an intensity variation to determine $f$, images with different $\varphi_{2 \mathrm{D}}$ thresholds can be compared to the original image.

Figure D1a shows the original image, whereas image D1b shows pixels which are equal to one. As it can be noticed, not all particles are seen in the image, meaning that the rest of particles have higher intensities than the minima values that have been registered in those pixels. Thus, we understand that the particle intensity fluctuates due to difference in the color tone.

In image D1c, all pixels which are above of $\varphi_{2 \mathrm{D}}=0.9$ are shown. In this case the clusters appear in the image, while not all particles that are isolated are captured in the image. It must be noticed that a single particle may not fully cover a single pixel if this is partly distributed over few pixels. Therefore, we consider that a too high $f$ value will definitely show all particles in the system, whereas a too low one will not show the cluster areas. Thus, we consider a that the suitable $f$ value should between those corresponding to images D1d, e

Figure D1f illustrates that at $\varphi_{2 D}>0.6$, almost all the particles are captured in the image, slightly over sizing the cluster areas. Thus, we can state that, in our experiment the particle intensity fluctuates between $\varphi_{2 D}=0.6$ and $\varphi_{2 D}=1$, obtaining an $f$ value of 0.3 .

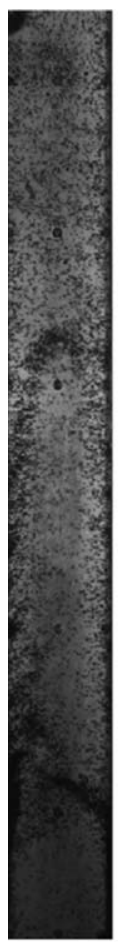

a

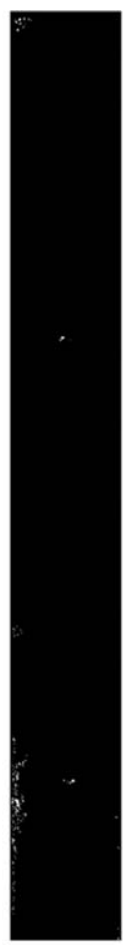

b

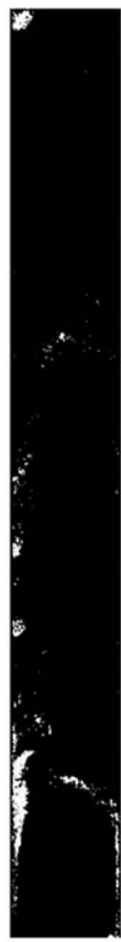

c

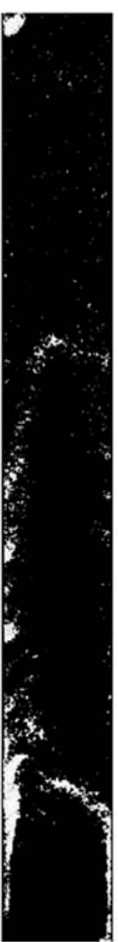

d

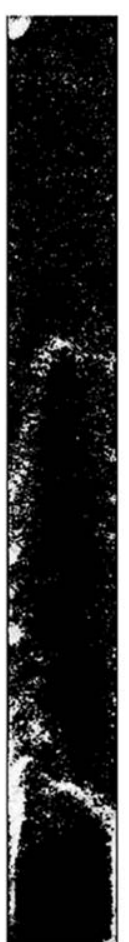

$\mathrm{e}$

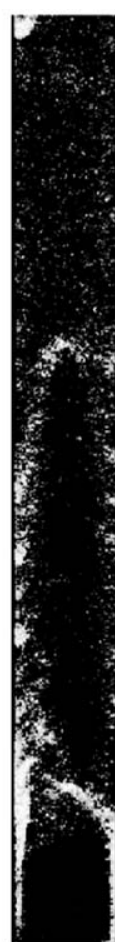

f
Figure D1. (a) Original image. (b) $\varphi_{2 D}=1$. (c) $\varphi_{2 D}>0.9$. (d) $\varphi_{2 D}>0.8$. (e) $\varphi_{2 D}>0.7$. (f) $\varphi_{2 D}>0.6$.

After evaluating the influence of this particle intensity fluctuations, we have fitted the dependence of $A$ and $B$ parameters into the equations below:

$$
\begin{gathered}
A\left(d_{p} / D\right)=0.6818 \cdot d_{p} / D+0.081 \cdot f+0.024 \pm 0.001 \\
B=0.99-0.45 \cdot f \pm 0.01
\end{gathered}
$$

It has to be noted that under strong changes of $f$, the profiles of $\left\langle\varphi_{3 D}\right\rangle$ are qualitatively the same, but slightly vary in terms of magnitude. In one extreme, where $f=0.4$, the time-averaged solids fraction of this experiment would be of $\left\langle\varphi_{3 D}\right\rangle=0.04$, while it would be of $\left\langle\varphi_{3 D}\right\rangle=0.03$ if $f$ is equal to 0 .

Manuscript received Aug. 13, 2015, and revision received Dec. 4, 2015. 